HIAS-E-27

\title{
Impulse Response Matching Estimators for DSGE Models
}

\author{
Pablo Guerron-Quintana \\ Federal Reserve Bank of Philadelphia, Philadelphia, PA 19106-1574
}

Atsushi Inoue

Department of Economics, Vanderbilt University, Nashville, TN 37235-1819 Hitotsubashi Institute for Advanced Study, Hitotsubashi University, 2-1 Naka, Kunitachi, Tokyo, Japan 186-8601

Lutz Kilian

Department of Economics, 238 Lorch Hall, Ann Arbor, MI 48109-1220

May 30, 2016

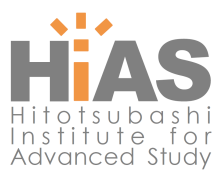

Hitotsubashi Institute for Advanced Study, Hitotsubashi University 2-1, Naka, Kunitachi, Tokyo 186-8601, Japan tel:+81 $425808604 \quad$ http://hias.ad.hit-u.ac.jp/

HIAS discussion papers can be downloaded without charge from: http://hdl.handle.net/10086/27202

https://ideas.repec.org/s/hit/hiasdp.html

All rights reserved. 


\title{
Impulse Response Matching Estimators for DSGE Models
}

\author{
Pablo Guerron-Quintana* \\ Federal Reserve Bank
of Philadelphia
}

Atsushi Inoue ${ }^{\dagger}$

Lutz Kilian

\author{
Vanderbilt University \\ Hitotsubashi Institute \\ for Advanced Study \\ Hitotsubashi University
}

May 30, 2016

\begin{abstract}
One of the leading methods of estimating the structural parameters of DSGE models is the VAR-based impulse response matching estimator. The existing asympotic theory for this estimator does not cover situations in which the number of impulse response parameters exceeds the number of VAR model parameters. Situations in which this order condition is violated arise routinely in applied work. We establish the consistency of the impulse response matching estimator in this situation, we derive its asymptotic distribution, and we show how this distribution can be approximated by bootstrap methods. Our analysis sheds new light on the choice of the weighting matrix and covers both weakly and strongly identified DSGE model parameters. We also show that under our assumptions special care is needed to ensure the asymptotic validity of Bayesian methods of inference. A simulation study suggests that the interval estimators we propose are reasonably accurate in practice. We also show that using these methods may affect the substantive conclusions in empirical work.
\end{abstract}

JEL Classification Number: C32, C52, E30, E50

Key Words: Structural estimation, DSGE, VAR, impulse response, nonstandard asymptotics, bootstrap, weak identification, robust inference.

\footnotetext{
${ }^{*}$ Federal Reserve Bank of Philadelphia, Philadelphia, PA 19106-1574. Email: pguerron@gmail.com.

${ }^{\dagger}$ Department of Economics, Vanderbilt University, Nashville, TN 37235-1819. E-mail: atsushi.inoue@vanderbilt.edu

${ }^{\ddagger}$ University of Michigan, Department of Economics, 238 Lorch Hall, Ann Arbor, MI 48109-1220. E-mail: lkilian@umich.edu.
} 


\section{Introduction}

Structural impulse responses play a central role in modern macroeconomics. It is common to estimate the structural parameters of a dynamic stochastic general equilibrium (DSGE) model by choosing these parameters so as to minimize a suitably weighted average of the distance between the structural impulse responses implied by the DSGE model and the corresponding structural impulse responses implied by an approximating vector autoregressive (VAR) model fit to actual data. One advantage of this approach compared with full information maximum likelihood estimators of DSGE models is that it does not require the model to fit well in all dimensions, but allows the user to focus on the dimension of the model that matters most to macroeconomists (also see Dridi, Guay and Renault 2007; Hall, Inoue, Nason, and Rossi 2012).

Such impulse response matching estimators have been employed in Rotemberg and Woodford (1997), Christiano, Eichenbaum and Evans (2005), Iacoviello (2005), Boivin and Giannoni (2006), Uribe and Yue (2006), DiCecio and Nelson (2007), Dupor, Han and Tsai (2007), Jordà and Kozicki (2007), DiCecio (2009), and Altig, Christiano, Eichenbaum and Lindé (2011), among others. In related research, Christiano, Trabandt and Walentin (2011) propose a Bayesian version of the impulse response matching estimator in which the quasi-likelihood function based on the distance between VAR and DSGE model impulse responses is combined with prior information. Other applications of Bayesian impulse response matching estimators include Christiano, Eichenbaum and Trabandt (2015) and Kormilitsina and Nekipelov (2016).

Because impulse response matching estimators are classical minimum distance (CMD) estimators, by construction they inherit the usual properties of CMD estimators (see, e.g., Newey and Smith 2004). Notably, the use of the optimal weighting matrix induces finitesample bias in the estimator, which is why most applied users employ a diagonal weighting matrix instead. In this paper we identify another potential problem that is specific to impulse response matching estimators. In estimating the structural parameters of DSGE 
models, macroeconomists often match response functions evaluated across many horizons such that the number of impulse response coefficients exceeds the dimensionality of the VAR model parameters (see, e.g., Iacoviello 2005; Uribe and Yue 2006; Altig, Christiano, Eichenbaum and Lindé 2011). This practice causes the joint distribution of the structural impulse responses to be singular, which in turn renders the asymptotic behavior of the resulting impulse response matching estimator nonstandard. As a result, standard asymptotic and finite-sample results for CMD estimators no longer apply. We develop an alternative asymptotic theory of the impulse response matching estimator for this practically relevant context. Our paper makes four distinct theoretical contributions.

First, we show that in this case the impulse response matching estimator has a nonstandard convergence rate when using the optimal weighting matrix. While the estimator remains consistent, its asymptotic distribution is nonstandard. Both the rate of convergence and the nonnormality of the asymptotic distribution differ from standard results for CMD estimators. We establish that the nonstandard asymptotic approximation may be recovered by bootstrap methods. Of course, in the absence of asymptotic normality, one would not want to report standard errors for this estimator, but rely on bootstrap confidence intervals that do not rely on asymptotic normality.

In contrast, the impulse response matching estimator based on the diagonal weighting matrix remains $\sqrt{T}$-consistent and asymptotically normal as in the standard CMD case. Its asymptotic variance may be approximated by the same bootstrap methods as in the case in which the dimensionality of the impulse response vector is no larger than that of the VAR model parameters. The latter result provides a formal justification for the use of the diagonal weighting matrix in applied work in a case not covered by existing asymptotic theory.

Second, our asymptotic results matter not only for the construction of point and interval estimates for structural parameters. We also prove that conventional tests of overidentifying restrictions, as employed in Boivin and Giannoni (2006), for example, have a nonstandard 
asymptotic distribution, when the number of impulse response parameters exceeds the number of VAR model parameters, invalidating the use of conventional critical values.

Third, our work also has implications for the use of Bayesian impulse response matching estimators. Often in the literature, Bayesian estimators are used as a convenient device for constructing asymptotic approximations. It may be tempting to base inference on a point estimate constructed from the mean, median or mode of the quasi-posterior of the structural parameters together with an estimate of the asymptotic standard error based on the standard deviation of this distribution. Although Markov Chain Monte Carlo methods may indeed be used to construct point estimators of the structural parameters based on the mean, median or mode, we show that one cannot use the standard deviations of the quasi-posterior distribution to approximate the asymptotic standard errors of the structural parameter estimator, when the number of impulse responses exceeds the number of VAR model parameters. This is true whether one employs the optimal weighting matrix or the diagonal weighting matrix. We show that asymptotically valid Bayesian inference may be conducted by constructing the variance using the sandwich formula of Chernozhukov and Hong (2003), however.

Fourth, it is well known that structural parameters of macroeconomic models may not be strongly identified. This problem also afflicts impulse response matching estimators, as documented in Canova and Sala (2009). We propose a nonstandard confidence interval for the structural parameters of the underlying data generating process that is robust to weak identification problems.

The remainder of the paper is organized as follows. Section 2 establishes the consistency of the impulse response matching estimators in question, derives their asymptotic distribution, and proposes suitable bootstrap methods of inference. Because both the impulse response matching estimator based on the optimal weighting matrix and the estimator based on the diagonal weighting matrix are practically feasible and asymptotically valid, the question arises which approach implies more accurate confidence intervals for structural 
model parameters in finite samples.

Section 3 evaluates the quality of these asymptotic approximations based on a Monte Carlo simulation experiment. Based on a small-scale New Keynesian model we provide some tentative evidence that confidence intervals for structural DSGE model parameters based on the diagonal weighting matrix tend to be more accurate than intervals based on the optimal weighting matrix. They also appear more robust to the choice of the VAR lag order and to the maximum horizon of the impulse response function. Satisfactory coverage accuracy, however, may require fairly large sample sizes, even when using the diagonal weighting matrix. The coverage deficiencies in small samples can be traced to approximation error in the VAR representation of the DSGE model. When bootstrapping the state-space representation of the DSGE model directly rather than its VAR approximation, high coverage accuracy is obtained even in small samples, albeit at the cost of taking a stand on the parametric structure of the data generating process. These baseline simulation results pertain to strongly identified structural DSGE model parameters. We also provide simulation evidence that VAR-based bootstrap confidence intervals that allow for weak identification tend to be reasonably accurate even in realistically small samples and are not systematically less accurate than their DSGE model bootstrap counterparts.

In Section 4, we illustrate the implementation of the proposed methods in the context of a prototypical medium-scale New Keynesian DSGE model of the type used at many central banks. This empirical example illustrates that basing estimates of the asymptotic standard error on the standard deviation of the quasi-posterior of the structural parameters results in much lower standard error estimates than the alternative estimation methods developed in this paper. For example, whereas the point estimate of the price-markup factor is quite robust to the choice of method, its standard error is about three times as large one would have concluded based on the standard deviation of the quasi-posterior. These results are based on the conventional premise in empirical work that the structural parameters are strongly identified. We also present alternative estimates that take account of the 
possibility that some parameters are only weakly identified. We illustrate that allowing for weak identification in some cases affects the substantive conclusions, while in others it does not. The concluding remarks are in Section 5. The proofs are contained in the Appendix A.

\section{Asymptotic Theory}

The thought experiment is that the data are generated by a DSGE model. At least some of the structural parameters of this DSGE model are unknown. The DSGE model is approximated by a finite-order structural VAR model with identifying restrictions that are consistent with the underlying DSGE model. ${ }^{1}$ The objective is to recover an estimate of the unknown structural parameters in the DSGE model by searching the space of these parameters for the parameter values that result in the closest match between the structural VAR impulse responses based on the actual data and those from the DSGE model evaluated at the hypothesized parameter values. We are concerned with the asymptotic properties of this impulse response matching estimator in repeated sampling. As is standard in this literature, it is assumed that the structural impulse responses obtained from the VAR model are strongly identified.

Let $\gamma_{0}$ denote the $q \times 1$ vector of the population structural impulse responses (excluding all impulse responses that are not estimated). Then the structural impulse response estimator $\widehat{\gamma}_{T}$ of $\gamma_{0}$ is obtained from an estimated VAR model fitted to the actual data, its bootstrap analogue $\widehat{\gamma}_{T}^{*}$ is obtained from a VAR model fitted to data simulated from the estimated VAR model, and the double-bootstrap estimator $\widehat{\gamma}_{T}^{* *}$ is obtained from a VAR model fitted to data simulated from the VAR model evaluated at the bootstrap parameter estimates. Closed-form solutions for the structural impulse response estimator from VAR models are provided in Lütkepohl (1990). Because structural impulse responses are func-

\footnotetext{
${ }^{1}$ Fernandez-Villaverde, Rubio-Ramirez, Sargent and Watson (2007) make precise the conditions under which a DSGE model may be approximated by a finite-order VAR model.
} 
tions of the slope parameters and of the error covariance matrix of the VAR model, which in turn are an implicit function of the first and second moments of the data, we can write $\gamma_{0}=\gamma(\mu), \widehat{\gamma}_{T}=\gamma\left(\bar{X}_{T}\right), \widehat{\gamma}_{T}^{*}=\gamma\left(\bar{X}_{T}^{*}\right)$ and $\widehat{\gamma}_{T}^{* *}=\gamma\left(\bar{X}_{T}^{* *}\right)$ where $\mu, \bar{X}_{T}, \bar{X}_{T}^{*}$ and $\bar{X}_{T}^{* *}$ are $k \times 1$ vectors of the population moments of the data, the sample moments, the bootstrap sample moments and the double-bootstrap sample moments, respectively. $k$ corresponds to the number of VAR model parameters, defined as the total number of slope parameters plus the number of unique elements in the error covariance matrix.

In impulse response matching estimation, an $l \times 1$ vector of structural parameters of a macroeconomic model, $\theta$, is estimated based on a restriction of the form:

$$
\gamma_{0}=f\left(\theta_{0}\right)
$$

In this paper, we consider two types of impulse response matching estimators. One is based on the optimal weighting matrix,

$$
\widehat{\theta}_{o p t, T}=\operatorname{argmin}_{\theta \in \Theta}\left(\widehat{\gamma}_{T}-f(\theta)\right)^{\prime} \widehat{\Sigma}_{T}^{*-1}\left(\widehat{\gamma}_{T}-f(\theta)\right),
$$

where $\widehat{\Sigma}_{T}^{*}$ is the bootstrap covariance matrix estimator of impulse responses,

$$
\widehat{\Sigma}_{T}^{*}=\frac{1}{B} \sum_{b=1}^{B}\left(\widehat{\gamma}_{T}^{*(b)}-\overline{\widehat{\gamma}}_{T}^{*}\right)\left(\widehat{\gamma}_{T}^{*(b)}-\overline{\widehat{\gamma}}_{T}^{*}\right)^{\prime}
$$

$\widehat{\gamma}_{T}^{*(b)}$ is the $b$ th bootstrap estimator of impulse responses for $b=1,2, \ldots, B$, and $\bar{\gamma}_{T}^{*}=$ $(1 / B) \sum_{b=1}^{B} \widehat{\gamma}^{*(b)}$. The other is

$$
\widehat{\theta}_{\text {diag }, T}=\operatorname{argmin}_{\theta \in \Theta}\left(\widehat{\gamma}_{T}-f(\theta)\right)^{\prime} W_{T}\left(\widehat{\gamma}_{T}-f(\theta)\right)
$$

where $W_{T}$ is a positive definite diagonal matrix, whose diagonal elements are the reciprocals of the diagonal elements of $\widehat{\Sigma}_{T}^{*}$. The bootstrap version of the impulse response matching 
estimators, $\widehat{\theta}_{\text {opt }, T}^{*}$ and $\widehat{\theta}_{\text {diag }, T}^{*}$, is defined in equations (2) and (4) with $\widehat{\gamma}_{T}$ and $\widehat{\Sigma}_{T}^{*}$ replaced by $\widehat{\gamma}_{T}^{*(b)}$ and $\widehat{\Sigma}_{T}^{* *}$. We refer to the bootstrap that is used to generate $\widehat{\gamma}_{T}^{*(b)}$ as the bootstrap. The bootstrap version of $\widehat{\theta}_{\text {opt }, T}$ and $\widehat{\theta}_{\text {diag,T }}$ is obtained by bootstrapping the realizations of the bootstrap data and calculating $\widehat{\gamma}_{T}^{* *}$ and their covariance matrix $\widehat{\Sigma}_{T}^{* *}$. We refer to this bootstrap as the double bootstrap.

\subsection{Asymptotic Properties of Impulse Response Matching Esti- mators of Structural Model Parameters}

We initially focus on a situation in which the structural parameters are strongly identified. Our results are based on stochastic expansions and matrix decompositions. Suppose that there are conformable matrices $B_{0}, \ldots, B_{H}$ such that

$$
\begin{aligned}
T^{\frac{1}{2}}\left(\gamma\left(\bar{X}_{T}\right)-\gamma(\mu)\right)= & B_{0} Z_{T}+T^{-\frac{1}{2}} B_{1}\left(Z_{T} \otimes Z_{T}\right) \\
& +\cdots+T^{-\frac{H}{2}} B_{H}\left(Z_{T} \otimes \cdots \otimes Z_{T}\right)+o_{p}\left(T^{-\frac{H}{2}}\right),
\end{aligned}
$$

where $H$ is the maximum horizon of the impulse responses to be matched. Conventional delta-method asymptotics are based on the leading term of this expansion. If $q>k$, the first-order asymptotic variance, $B_{0} B_{0}^{\prime}$, will be singular. It follows from the Schur decomposition theorem (Theorem 13 of Magnus and Neudecker, 1999, p.16) that there exists an orthonormal matrix $\tilde{S}$ whose columns are eigenvectors of $B_{0} B_{0}^{\prime}$ and a diagonal matrix $\Lambda$ whose diagonal elements are the eigenvalues of $B_{0} B_{0}^{\prime}$ such that

$$
\tilde{S}^{\prime} B_{0} B_{0}^{\prime} \tilde{S}=\Lambda
$$

The stochastic expansion (5) and the decomposition (6) allow us to analyze the asymptotic behavior of the impulse response matching estimator when the number of impulse response parameters exceeds the number of VAR model parameters. 
To be precise, let $(\Omega, \mathcal{F}, P)$ denote the data probability space, $\left(\Psi, \mathcal{G}, P^{*}\right)$ the bootstrap probability measure, and $\left(\Phi, \mathcal{H}, P^{* *}\right)$ the double bootstrap probability measure. Let $X_{T}^{*}$ and $X^{*}\left[X_{T}^{* *}\right.$ and $\left.X^{* *}\right]$ denote a generic bootstrap statistic and a random variable that are defined on the product probability space $(\Omega, \mathcal{F}, P) \times\left(\Psi, \mathcal{G}, P^{*}\right)[(\Omega, \mathcal{F}, P) \times(\Psi, \mathcal{G}, P) \times$ $\left.\left.\Phi, \mathcal{H}, P^{* *}\right)\right]$, respectively. Following Dovonon and Gonçalves (2014), we define:

(i) $X_{T}^{*}=o_{p}^{*}(1)$ in prob- $P$ if for any $\varepsilon, \delta>0, P\left(P^{*}\left(\left|X_{T}^{*}\right|>\varepsilon\right)>\delta\right) \rightarrow 0$ as $T \rightarrow \infty$.

(ii) $X_{T}^{*}=O_{p}^{*}(1)$ in prob- $P$ if for any $\delta>0$ there exists $0<M<\infty$ such that $P\left(P^{*}\left(\left|X_{T}^{*}\right| \geq M\right)>\delta\right) \rightarrow 0$ as $T \rightarrow \infty$.

(iii) $X_{T}^{* *}=o_{p}^{* *}(1)$ in prob- $P^{*}$ if for any $\varepsilon, \delta>0, P^{*}\left(P^{* *}\left(\left|X_{T}^{* *}\right|>\varepsilon\right)>\delta\right) \rightarrow 0$ a.s. as $T \rightarrow \infty$.

(iv) $X_{T}^{* *}=O_{p}^{* *}(1)$ in prob- $P^{*}$ if for any $\delta>0$ there exists $0<M<\infty$ such that $P^{*}\left(P^{* *}\left(\left|X_{T}^{* *}\right| \geq M\right)>\delta\right) \rightarrow 0$ a.s. as $T \rightarrow \infty$.

(v) $X_{T}^{*} \stackrel{d^{*}}{\rightarrow} X^{*}$ in prob- $P$ if $E^{*} f\left(X_{T}^{*}\right) \rightarrow E\left(f\left(X^{*}\right)\right)$ in prob- $P$ for every continuous and bounded function $f$, where $E^{*}(\cdot)$ is the expectation operator with respect to the bootstrap probability measure conditional on data.

(vi) $X_{T}^{* *} \stackrel{d^{* *}}{\rightarrow} X^{* *}$ in prob- $P^{*}$ if $E^{* *} f\left(X_{T}^{* *}\right) \rightarrow E^{*}\left(f\left(X^{* *}\right)\right)$ in prob- $P^{*}$ a.s. for every continuous and bounded function $f$, where $E^{* *}(\cdot)$ is the expectation operator with respect to the double bootstrap probability measure conditional on a bootstrap realization and the data.

Our analysis requires the following conditions. 


\section{Assumptions.}

(a) $Z_{T} \equiv \Omega^{-\frac{1}{2}} \sqrt{T}\left(\bar{X}_{T}-\mu\right) \stackrel{d}{\rightarrow} Z \sim \mathcal{N}\left(0, I_{k}\right)$ and $Z_{T}^{*} \equiv \Omega^{-\frac{1}{2}} \sqrt{T}\left(\bar{X}_{T}^{*}-\bar{X}_{T}\right) \stackrel{d^{*}}{\rightarrow} Z^{*} \sim$ $\mathcal{N}\left(0, I_{k}\right)$ in prob- $P$ where $\Omega$ is a positive definite matrix.

(b) Given the maximum impulse response horizon $H$, there are conformable matrices $B_{0}, \ldots, B_{H}$ and $\widehat{B}_{0}, \ldots, \widehat{B}_{H}$ such that

$$
\begin{aligned}
T^{\frac{1}{2}}\left(\gamma\left(\bar{X}_{T}\right)-\gamma(\mu)\right)= & B_{0} Z_{T}+T^{-\frac{1}{2}} B_{1}\left(Z_{T} \otimes Z_{T}\right) \\
& +\cdots+T^{-\frac{H}{2}} B_{H}\left(Z_{T} \otimes \cdots \otimes Z_{T}\right)+o_{p}\left(T^{-\frac{H}{2}}\right) \\
T^{\frac{1}{2}}\left(\gamma\left(\bar{X}_{T}^{*}\right)-\gamma\left(\bar{X}_{T}\right)\right)= & \widehat{B}_{0} Z_{T}^{*}+T^{-\frac{1}{2}} \widehat{B}_{1}\left(Z_{T}^{*} \otimes Z_{T}^{*}\right) \\
& +\cdots+T^{-\frac{H}{2}} \widehat{B}_{H}\left(Z_{T}^{*} \otimes \cdots \otimes Z_{T}^{*}\right)+o_{p}^{*}\left(T^{-\frac{H}{2}}\right) \text { in prob- } P(8)
\end{aligned}
$$

where $\widehat{B}_{j}=B_{j}+o_{p}(1)$ for $j=0,1, \ldots, H$.

(c) Recall the orthonormal matrix $\widetilde{S}$ defined by $\tilde{S}^{\prime} B_{0} B_{0}^{\prime} \tilde{S}=\Lambda$. We interchange the columns of $\widetilde{S}$ such that $S=\left[\begin{array}{lllll}S_{0} & S_{1} & S_{2} & \cdots & S_{r}\end{array}\right]$, where $S_{0}$ is the $q \times q_{0}$ matrix that consists of the eigenvectors associated with the $k$ largest eigenvalues of $B_{0} B_{0}^{\prime}$, and the matrices $S_{1}, S_{2}, \ldots, S_{r}$ are of dimension $q \times q_{1}, q \times q_{2}, \ldots, q \times q_{r}$, respectively, such that $q_{0}=k$ and $q_{0}+q_{1}+\ldots+q_{r}=q$. Let $S_{0}, S_{1}, \ldots, S_{r}$ satisfy the conditions: (i) $S_{i}^{\prime} B_{j}=0$ for $\forall i=1, \ldots, r$ and $\forall j<j_{i}$; and (ii)

$$
\xi=\left[\begin{array}{c}
S_{0}^{\prime} B_{0} Z \\
S_{1}^{\prime} B_{j_{1}}(Z \otimes \cdots \otimes Z) \\
\vdots \\
S_{r}^{\prime} B_{j_{r}}(Z \otimes \cdots \otimes Z)
\end{array}\right]
$$

has a nonsingular second moment matrix $J=E\left(\xi \xi^{\prime}\right)$, where $j_{1}, j_{2}, \ldots, j_{r}$ are integers such that $0<j_{1}<j_{2}<\ldots<j_{r}$.

(d) $\widehat{S}-S=o_{p}(1), \widehat{S}^{*}-\widehat{S}=o_{p}^{*}(1)$ in prob- $P$ and $\widehat{S}^{* *}-\widehat{S}^{*}=o_{p}^{* *}(1)$ in prob- $P^{*}$ where $\widehat{S}$, 
$\widehat{S}^{*}$ and $\widehat{S}^{* *}$ are the sample, bootstrap and double bootstrap analogues of $S$.

(e)

$$
\Upsilon_{T} S^{\prime}\left(\gamma\left(\bar{X}_{T}^{*}\right)-\gamma\left(\bar{X}_{T}\right)\right)\left(\gamma\left(\bar{X}_{T}^{*}\right)-\gamma\left(\bar{X}_{T}\right)\right)^{\prime} S \Upsilon_{T}
$$

and

$$
\Upsilon_{T} S^{\prime}\left(\gamma\left(\bar{X}_{T}^{* *}\right)-\gamma\left(\bar{X}_{T}^{*}\right)\right)\left(\gamma\left(\bar{X}_{T}^{* *}\right)-\gamma\left(\bar{X}_{T}^{*}\right)\right)^{\prime} S \Upsilon_{T}
$$

are uniformly integrable with respect to the bootstrap and the double bootstrap probability measures, respectively, conditional on the data, where $S$ has been defined in Assumption (c) and where

$$
\Upsilon_{T}=\left[\begin{array}{cccc}
T^{\frac{1}{2}} I_{q_{0}} & 0_{q_{0} \times q_{1}} & \cdots & 0_{q_{0} \times q_{r}} \\
0_{q_{1} \times q_{0}} & T^{\frac{j_{1}+1}{2}} I_{q_{1}} & \cdots & 0_{q_{1} \times q_{r}} \\
\vdots & \vdots & \ddots & \vdots \\
0_{q_{r} \times q_{0}} & 0_{q_{r} \times q_{1}} & \cdots & T^{\frac{j_{r}+1}{2}} I_{q_{r}}
\end{array}\right]
$$

(f) There is a unique value of $\theta, \theta_{0}$, in the interior of a compact set in $\Re^{l}, \Theta$, such that $\gamma(\mu)=f\left(\theta_{0}\right)$, where $f: \Theta \rightarrow \Re^{q}$ is continuously differentiable in $\theta$ in the interior of $\Theta$. The Jacobian matrix of $f$ at $\theta_{0}, F_{0}=F\left(\theta_{0}\right)$, where $F(\theta)=\partial f(\theta) / \partial \theta^{\prime}$, has rank $l$ and $l$ is the dimension of $\theta$.

(g) $W_{T}, W_{T}^{*}$ and $W_{T}^{* *}$ are sequences of positive definite matrices such that $W_{T}=W+$ $o_{p}(1), W_{T}^{*}=W_{T}+o_{p}^{*}(1)$ in prob- $P$, and $W_{T}^{* *}=W_{T}^{*}+o_{p}^{* *}$ in prob- $P^{*}$ where $W$ is positive definite.

(h) (1) For the optimal weighting matrix, if $q>k$, then $\theta_{0}$ is the unique value of $\theta$ in $\Theta$ that satisfies $S_{r}^{\prime}\left(f\left(\theta_{0}\right)-f(\theta)\right)=0_{q_{r} \times 1}$ where $S_{r}$ is the $q \times q_{r}$ submatrix of $S$ that consists of the last $q_{r}$ columns of $S$ as defined in the proof of Theorem 1.

(2) If $W_{T}$ is a diagonal weighting matrix, $F_{0}^{\prime} W B_{0}$ has rank $l$. 
Remarks. 1. Assumption (a) holds, for example, when applying the residual-based bootstrap to stationary homoskedastic vector autoregressive processes.

2. Assumption (b) follows from a Taylor series expansion of the left-hand side of equations (7) and (8). The delta method is based on the first-order term of the stochastic expansion on the right-hand side. The higher-order stochastic terms on the right-hand side have also been used to develop Edgeworth expansions of the distribution of estimators (see Hall 1992). Assumption (b) holds, for example, for stationary vector autoregressive processes with positive definite error covariance matrices and short-run exclusion restrictions. For more primitive assumptions for the existence of asymptotic expansions of the distribution of estimators in stationary time series models see Bao and Ullah (2007) and Bao (2007). The precise specification of the expansions (7) and (8) changes with the specification of the VAR model and with the choice of the maximum horizon $H$. The $B_{j}$ matrices can be expressed as functions of the VAR model parameters, allowing their consistent estimation under standard assumptions. For the implementation of the impulse response matching estimator proposed in this section, no explicit derivation or estimation of the expansions (7) and (8) is required. All we require is their existence.

3. Assumption (c) is a high-level assumption, but may in principle be verified. Two fully worked out examples can be found in Appendix B. In larger models establishing the nonsingularity of $J$ may necessitate the use of numerical derivatives. When $r=2$, a failure of the rank condition for $J$ may be detected by checking the order condition that $q_{1} \leq k^{2}$. This follows from the fact that, if $S=\left[S_{0} S_{1}\right]$ and $B_{1}$ is of full rank,

$J=\left[\begin{array}{cc}S_{0}^{\prime} B_{0} B_{0}^{\prime} S_{0} & 0_{q_{0} \times q_{1}} \\ 0_{q_{1} \times q_{0}} & S_{1}^{\prime} B_{1} E\left(Z Z^{\prime} \otimes Z Z^{\prime}\right) B_{1}^{\prime} S_{1}\end{array}\right]=\left[\begin{array}{cc}\Lambda_{0} & 0_{q_{0} \times q_{1}} \\ 0_{q_{1} \times q_{0}} & S_{1}^{\prime} B_{1} E\left(Z Z^{\prime} \otimes Z Z^{\prime}\right) B_{1}^{\prime} S_{1},\end{array}\right]$,

where $\Lambda_{0}$ is the $q_{0} \times q_{0}$ diagonal matrix whose diagonal elements are given by the nonzero eigenvalues of $B_{0} B_{0}^{\prime}$. When $B_{1}$ has full rank, $S_{1}^{\prime} B_{1}$ also has full rank because $S$ is orthogonal and thus $S_{1}$ has rank $q_{1}$. 
4. Assumption (d) rules out cases in which the eigenvectors are discontinuous in the data, even though eigenvalues are not (see Dufour and Valery 2015).

5. Assumption (e) ensures that the bootstrap method can be used to estimate the limiting covariance matrix.

6. Assumptions (f) and (g) are standard assumptions for classical minimum distance estimators.

7. Assumption $(\mathrm{h})(1)$ is for the impulse response matching estimator based on the optimal weighting matrix and is stronger than the identification condition in Assumption (f). Because $q_{r}<q$ can be smaller than $l$, there may be another value of $\theta$ at which $\gamma(\mu)-f(\theta)=f\left(\theta_{0}\right)-f(\theta) \neq 0_{q \times 1}$ and $S_{r}^{\prime}\left(f\left(\theta_{0}\right)-f(\theta)\right)=0_{q_{r} \times 1}$.

8. Assumption (h)(2) is for the impulse response matching estimator based on the diagonal weighting matrix. When $\operatorname{rank}\left(B_{0}\right)=q$, it simplifies to the standard assumption that the Jacobian has full rank, i.e., $\operatorname{rank}\left(F\left(\theta_{0}\right)\right)=l$.

Theorem 1 (Consistency of Impulse Response Matching Estimators). Suppose that Assumptions (a)-(h) hold. Then

(a) $\widehat{\theta}_{o p t, T}=\theta_{0}+o_{p}^{*}(1)$ in prob- $P$.

(b) $\widehat{\theta}_{\text {diag }, T}=\theta_{0}+o_{p}^{* *}(1)$ in prob- $P^{*}$.

Next, we derive the asymptotic distributions of the impulse response matching estimator.

Theorem 2 (Asymptotic Distributions of Impulse Response Matching Estimators). Suppose that Assumptions (a)-(h) hold. Then

(a)

$$
\begin{aligned}
& T^{\frac{j_{r}+1}{2}}\left(\widehat{\theta}_{o p t, T}-\theta_{0}\right) \stackrel{d^{*}}{\rightarrow}\left(F_{0}^{\prime} S_{r} \overline{\bar{J}} S_{r}^{\prime} F_{0}\right)^{-1} F_{0}^{\prime} S_{r} \bar{J} \xi \text { in prob- } P, \\
& T^{\frac{j_{r}+1}{2}}\left(\widehat{\theta}_{o p t, T}^{*}-\widehat{\theta}_{o p t, T}\right) \stackrel{d^{* *}}{\rightarrow}\left(F_{0}^{\prime} S_{r} \overline{\bar{J}} S_{r}^{\prime} F_{0}\right)^{-1} F_{0}^{\prime} S_{r} \bar{J} \xi^{*} \text { in prob- } P^{*},
\end{aligned}
$$


where $r$ is defined in Assumption (c), $\overline{\bar{J}}$ is the $q_{r} \times q_{r}$ lower-right submatrix of $J^{-1}$ and $\bar{J}$ is the $q_{r} \times q$ lower submatrix of $J^{-1}$, and

(b)

$$
\begin{aligned}
T^{\frac{1}{2}}\left(\widehat{\theta}_{\text {diag }, T}-\theta_{0}\right) & \stackrel{d^{*}}{\rightarrow}\left(F_{0}^{\prime} W F_{0}\right)^{-1} F_{0}^{\prime} W B_{0} Z \text { in prob- } P, \\
T^{\frac{1}{2}}\left(\widehat{\theta}_{\text {diag }, T}^{*}-\widehat{\theta}_{\text {diag }, T}\right) & \stackrel{d^{* *}}{\rightarrow}\left(F_{0}^{\prime} W F_{0}\right)^{-1} F_{0}^{\prime} W B_{0} Z^{*} \text { in prob- } P^{*}
\end{aligned}
$$

Here the convergences in (10) and (12) are with respect to the bootstrap probability measure conditional on the data.

Remarks. Theorem 2(a) shows that the impulse response matching estimator based on the optimal weighting matrix has a nonstandard convergence rate that is faster than $T^{\frac{1}{2}}$ and has a nonstandard asymptotic distribution, when the number of impulse responses exceeds the number of VAR model parameters. Although the bootstrap can mimic the convergence rate and nonstandard asymptotic distribution, $\hat{\theta}_{\text {opt }, T}$ has higher-order bias in that the limiting distribution has nonzero mean if $\xi$ involves even powers of $Z$. This fact makes it necessary to employ bootstrap confidence intervals that can accommodate these features such as Hall's percentile interval. In contrast the impulse response matching estimator based on the diagonal weighting matrix in Theorem 2(b) is $\sqrt{T}$-consistent and asymptotically normal because $Z$ and $Z^{*}$ are normal.

\subsection{Asymptotic Distributions of the Test Statistic for Overiden- tifying Restrictions}

The results of section 2.1 not only have implications for the construction of point and interval estimates of the structural parameters, but they also affect tests of overidentifying restrictions. The conventional test statistic for overidentifying restrictions for testing the 
null hypothesis that $\gamma(\mu)=f\left(\theta_{0}\right)$ is defined as

$$
J_{T}=\left(\widehat{\gamma}_{T}-f\left(\widehat{\theta}_{o p t, T}\right)\right)^{\prime} \widehat{\Sigma}_{T}^{*-1}\left(\widehat{\gamma}_{T}-f\left(\widehat{\theta}_{o p t, T}\right)\right) .
$$

Under standard assumptions including $q \leq k$, the test statistic has an asymptotic $\chi^{2}$ distribution under the null hypothesis. This test has been used, for example, in Boivin and Giannoni (2006). The bootstrap analogue of this test statistic is defined as

$$
J_{T}^{*}=\left(\widehat{\gamma}_{T}^{*}-f\left(\widehat{\theta}_{o p t, T}^{*}\right)-\widehat{\gamma}_{T}+f\left(\widehat{\theta}_{o p t, T}\right)\right)^{\prime} \widehat{\Sigma}_{T}^{* *-1}\left(\widehat{\gamma}_{T}^{*}-f\left(\widehat{\theta}_{o p t, T}^{*}\right)-\widehat{\gamma}_{T}+f\left(\widehat{\theta}_{o p t, T}\right)\right)
$$

where the term $\widehat{\gamma}_{T}-f\left(\widehat{\theta}_{\text {opt }, T}\right)$ accomplishes the required recentering (see Hall and Horowitz 1996).

Theorem 3 (Asymptotic Distribution of the Test Statistic for Overidentifying Restrictions). Suppose that Assumptions (a)-(g) and (h)(1) hold. Then

$$
\begin{array}{ll}
J_{T} & \stackrel{d^{*}}{\rightarrow} \eta^{\prime} \eta \text { in prob- } P, \\
J_{T}^{*} \stackrel{d^{* *}}{\rightarrow} \eta^{* \prime} \eta^{*} \text { in prob- } P^{*},
\end{array}
$$

where

$$
\begin{aligned}
\eta & =J^{-\frac{1}{2}} \xi-J^{-\frac{1}{2}} S_{r}^{\prime} F_{0}\left(F_{0}^{\prime} S_{r} \overline{\bar{J}} S_{r}^{\prime} F_{0}\right)^{-1} F_{0}^{\prime} S_{r} \bar{J} \xi, \\
\eta^{*} & =J^{-\frac{1}{2}} \xi^{*}-J^{-\frac{1}{2}} S_{r}^{\prime} F_{0}\left(F_{0}^{\prime} S_{r} \overline{\bar{J}} S_{r}^{\prime} F_{0}\right)^{-1} F_{0}^{\prime} S_{r} \bar{J} \xi^{*} .
\end{aligned}
$$

Theorem 3 shows that the asymptotic distribution of the test statistic for overidentifying restrictions is nonstandard if $q>k$, but can be mimicked by the bootstrap. 


\subsection{Asymptotic Distributions of Bayesian Impulse Response Match- ing Estimators}

Next, we evaluate the Bayesian impulse response matching estimator of Christiano et al. (2011) and Christiano et al. (2015) from an asymptotic point of view. Christiano et al. motivate their approach as building on the analysis in Kim (2002) in particular. Define the quasi-posterior density as

$$
p(\theta)=\frac{\exp \left(-q_{T}(\theta)\right) \pi(\theta)}{\int_{\Theta} \exp \left(-q_{T}(\theta)\right) \pi(\theta) d \theta}
$$

where $\pi(\theta)$ is the prior density and

$$
q_{T}(\theta)=\frac{1}{2}\left(\widehat{\gamma}_{T}-f(\theta)\right)^{\prime} \widehat{\Sigma}_{T}^{*-1}\left(\widehat{\gamma}_{T}-f(\theta)\right)
$$

Under standard assumptions the quasi-posterior density converges to the asymptotic distribution of the impulse response matching estimator. As in Chernozhukov and Hong (2003), the quasi-posterior density is concentrated in a $T^{-\left(j_{r}+1\right) / 2}$ neighborhood around $\theta_{0}$, which is characterized by the local parameter

$$
h=T^{\frac{j_{r}+1}{2}}\left(\theta-\theta_{0}\right)+T^{\frac{j_{r}+1}{2}}\left(\nabla^{2} q_{T}\left(\theta_{0}\right)\right)^{-1} \nabla q_{T}\left(\theta_{0}\right),
$$

where $\nabla$ denotes the gradient and $\nabla^{2}$ the Hessian. $T^{\frac{j_{r}+1}{2}}$ is used because of the convergence rate of the impulse response matching estimator. Define the quasi-posterior density for $h$ as

$$
p_{T}^{*}(h)=T^{-\frac{l\left(j_{r}+1\right)}{2}} p_{T}\left(\frac{h}{T^{\frac{j_{r}+1}{2}}}+\theta_{0}-\left(\nabla^{2} q_{T}\left(\theta_{0}\right)\right)^{-1} \nabla q_{T}\left(\theta_{0}\right)\right)
$$

The next theorem establishes the asymptotic behavior of this quasi-posterior distribution.

Theorem 4 (Asymptotic Behavior of the Quasi-Posterior Distribution). Suppose that Assump- 
tions (a)-(g) and (h)(1) hold. Then, using the notation of Chernozhykov and Hong (2003),

$$
\left\|p_{T}^{*}(h)-p_{\infty}^{*}(h)\right\|_{T V M(\alpha)} \equiv \int_{H_{T}}\left(1+\|h\|^{\alpha}\right)\left|p_{T}^{*}(h)-p_{\infty}^{*}(h)\right| d h=o_{p}^{*}(1) \text { in prob- } P
$$

where

$$
\begin{aligned}
H_{T} & =\left\{h \in \Re^{l}: h=T^{\frac{j_{r}+1}{2}}\left(\theta-\theta_{0}\right)-T^{\frac{j_{r}+1}{2}}\left(\nabla^{2} q_{T}\left(\theta_{0}\right)\right)^{-1} \nabla q_{T}\left(\theta_{0}\right) \text { for some } \theta \in \Theta\right\}, \\
p_{\infty}^{*}(h) & =\sqrt{\frac{\left|T^{-\left(j_{r}+1\right)} \nabla^{2} q_{T}\left(\theta_{0}\right)\right|}{(2 \pi)^{l}}} \exp \left(-\frac{1}{2 T^{j_{r}+1}} h^{\prime} \nabla^{2} q_{T}\left(\theta_{0}\right) h\right) .
\end{aligned}
$$

Remarks. 1. Theorem 4 shows that the quasi-posterior is concentrated around the impulse response matching estimator $\widehat{\theta}_{T}$ and will be locally asymptotically normal. Heuristically, this follows from a quadratic expansion of $q_{T}(\theta)$ around $\widehat{\theta}_{T}$ in a neighborhood of $\widehat{\theta}_{T}$ :

$$
q_{T}(\theta) \approx q_{T}\left(\widehat{\theta}_{T}\right)+\frac{1}{2}\left(\theta-\widehat{\theta}_{T}\right)^{\prime} \nabla^{2} q_{T}\left(\widehat{\theta}_{T}\right)\left(\theta-\widehat{\theta}_{T}\right)
$$

where the linear term is zero due to the first-order condition for $\widehat{\theta}_{T}$. Because $q_{T}\left(\widehat{\theta}_{T}\right)$ is constant in $\theta$, the quasi-posterior is approximately proportional to

$$
\exp \left(-\frac{1}{2}\left(\theta-\widehat{\theta}_{T}\right)^{\prime} \nabla^{2} q_{T}\left(\widehat{\theta}_{T}\right)\left(\theta-\widehat{\theta}_{T}\right)\right)
$$

provided $\pi\left(\theta_{0}\right)>0$, which implies a normal density with mean $\widehat{\theta}_{T}$ and covariance matrix $\left[\nabla^{2} q_{T}\left(\widehat{\theta}_{T}\right)\right]^{-1}$.

2. This result means that the posterior distribution in equation (23) is different from the asymptotic distribution of the impulse response matching estimator in equation (11). Hence, Markov Chain Monte Carlo draws, which are designed to characterize the quasiposterior distribution, cannot be used to estimate asymptotic standard errors and confidence intervals when $q>k$. Under Assumption (h)(1), a necessary condition for estimating the asymptotic standard errors from the quasi-posterior is that $q \leq k$.

3. Whether $q>k$ or $q \leq k$, a consistent estimate of the asymptotic standard error under the 
diagonal weighting matrix may be obtained using the sandwich formular of Chernozhukov and Hong (2003, p. 307). Specifically, the asymptotic covariance may be estimated as

$$
\left(F_{0}^{\prime} W_{T} F_{0}\right)^{-1} F_{0}^{\prime} W_{T} \widehat{\Sigma}_{T}^{*} W_{T} F_{0}\left(F_{0}^{\prime} W_{T} F_{0}\right)^{-1}
$$

where $F_{0}$ is the Jacobian of the impulse response evaluated at $\theta_{0}$ and may be estimated by

numerical differentiation, $W_{T}$ is the diagonal weighting matrix, and $\widehat{\Sigma}_{T}^{*}$ is an estimate of the inverse of the optimal weighting matrix.

4. It can be shown that the same problem arises when Assumption (h)(1) is replaced by Assumption $(\mathrm{h})(2)$. In that case, the quasi-posterior again is normal, but different from equation (11), so we cannot rely on the standard errors of the quasi-posterior for asymptotic inference, but must evaluate the sandwich formula in Chernozhukov and Hong (2003).

\subsection{Inference When Identification is Not Strong}

A common problem in applied work is that some parameters of the DSGE model may not be strongly identified. In the GMM context, this problem was first discussed in Stock and Wright (2000). While several methods of inference have been developed that are robust to weak identification problems in DSGE models, none of these methods are designed for impulse response matching estimators (e.g., Guerron-Quintana, Inoue and Kilian 2013; Dufour, Khalaf and Kichian 2013; Qu 2014; Andrews and Mikusheva 2015).

Below we derive the asymptotic distribution of the Wald test statistic without assuming the identifiability of $\theta_{0}$. Our results apply whether $q>k$ or $q \leq k$.

Proposition (Asymptotic Distributions of the Wald Test Statistic of the Structural Impulse Responses).

Suppose that Assumptions (a)-(g) hold. Under $H_{0}: \gamma(\mu)=f\left(\theta_{0}\right)$ for some $\theta_{0} \in \Theta$, 
where $\theta_{0}$ need not be unique,

$$
\begin{aligned}
& \mathcal{W}_{T} \stackrel{d^{*}}{\rightarrow} \xi^{\prime} J^{-1} \xi \text { in prob- } P, \\
& \mathcal{W}_{T}^{*} \stackrel{d^{* *}}{\rightarrow} \xi^{* \prime} J^{-1} \xi^{*} \text { in prob- } P^{*},
\end{aligned}
$$

where

$$
\begin{aligned}
\mathcal{W}_{T} & =\left(\widehat{\gamma}_{T}-f\left(\theta_{0}\right)^{\prime} \Sigma_{T}^{*-1}\left(\widehat{\gamma}_{T}-f\left(\theta_{0}\right)\right),\right. \\
\mathcal{W}_{T}^{*} & =\left(\widehat{\gamma}_{T}^{*}-\widehat{\gamma}_{T}\right)^{\prime} \Sigma_{T}^{* *-1}\left(\widehat{\gamma}_{T}^{*}-\widehat{\gamma}_{T}\right), \\
\Sigma_{T}^{*} & =\frac{1}{B} \sum_{j=1}^{B}\left(\widehat{\gamma}_{T}^{*(j)}-\overline{\widehat{\gamma}}_{T}^{*}\right)\left(\widehat{\gamma}_{T}^{*(j)}-\overline{\widehat{\gamma}}_{T}^{*}\right)^{\prime}, \\
\Sigma_{T}^{* *(j)} & =\frac{1}{B} \sum_{k=1}^{B}\left(\widehat{\gamma}_{T}^{* *(j, k)}-\overline{\widehat{\gamma}}_{T}^{(j) * *}\right)\left(\widehat{\gamma}_{T}^{* *(j, k)}-\overline{\widehat{\gamma}}_{T}^{(j) * *}\right)^{\prime},
\end{aligned}
$$

with $\widehat{\gamma}_{T}^{* *(j, k)}=\gamma\left(\bar{X}_{T}^{* *(j, k)}\right)$ denoting the $k$ th bootstrap draw of the structural impulse response based on the $j$ th bootstrap estimate and $\bar{\gamma}_{T}^{* *(j)}=(1 / B) \sum_{k=1}^{B} \widehat{\gamma}_{T}^{* *(j)}$.

This proposition follows from Theorem 2 in Inoue and Kilian (2016) by replacing $\gamma_{0}=\gamma(\mu)$ with $f\left(\theta_{0}\right)$. Because the asymptotic distribution does not depend on the strength of the identification of $\theta_{0}$ and can be approximated by the bootstrap, one can invert the Wald statistic to obtain a $100(1-\varsigma) \%$ asymptotic confidence set for $\theta_{0}$ that is robust to weak identification,

$$
\left\{\theta \in \Theta: \mathcal{W}_{T}(\theta) \leq \mathcal{W}_{1-\varsigma}^{*}\right\}
$$

where $\mathcal{W}_{1-\varsigma}^{*}$ is the $100(1-\varsigma)$ percentile of the bootstrap distribution of $\mathcal{W}_{T}^{*}(\theta)$. Pointwise confidence intervals for the individual elements of the structural parameter vector $\theta$ may be obtained by the projection method (see, e.g., Dufour and Taamouti 2005, Chaudhuri and Zivot 2011; Guerron-Quintana, Inoue and Kilian 2013). 


\section{Monte Carlo Simulation Experiments}

For this section, we focus on a small-scale New Keynesian model that often serves as an illustrative example in the literature. This model consists of a Phillips curve, a Taylor rule, an investment-savings relationship, and the exogenous driving processes $z_{t}$ and $\xi_{t}$ :

$$
\begin{aligned}
\pi_{t} & =\kappa x_{t}+\beta \mathbb{E}\left(\pi_{t+1} \mid \mathcal{I}_{t-1}\right), \\
R_{t} & =\rho_{r} R_{t-1}+\left(1-\rho_{r}\right) \phi_{\pi} \pi_{t}+\left(1-\rho_{r}\right) \phi_{x} x_{t}+\xi_{t}, \\
x_{t} & =\mathbb{E}\left(x_{t+1} \mid \mathcal{I}_{t-1}\right)-\sigma\left(\mathbb{E}\left(R_{t} \mid \mathcal{I}_{t-1}\right)-\mathbb{E}\left(\pi_{t+1} \mid \mathcal{I}_{t-1}\right)-z_{t}\right), \\
z_{t} & =\rho_{z} z_{t-1}+\sigma^{z} \varepsilon_{t}^{z}, \\
\xi_{t} & =\sigma^{r} \varepsilon_{t}^{r},
\end{aligned}
$$

where $x_{t}, \pi_{t}$ and $R_{t}$ denote the output gap, inflation rate, and interest rate, respectively. Note that this model has more variables than shocks. The structural shocks $\varepsilon_{t}^{z}$ and $\varepsilon_{t}^{r}$ are assumed to be distributed $\mathcal{N} \mathcal{I D}(0,1)$. The model parameters are the discount factor $\beta$, the intertemporal elasticity of substitution $1 / \sigma$, the probability $\alpha$ of not adjusting prices for a given firm, the elasticity of substitution across varieties of goods, $\theta$, the parameter $\omega$ controlling disutility of labor supply; $\phi_{\pi}$ and $\phi_{x}$ capture the central bank's reaction to changes in inflation and the output gap, respectively, and $\kappa=\frac{(1-\alpha)(1-\alpha \beta)}{\alpha} \frac{\omega+\sigma}{\sigma(\omega+\theta)}$.

While this model is similar to the model used in Guerron-Quintana et al. (2013), there is one crucial difference. In this model, inflation and real output do not react contemporaneously to the monetary policy shock, $\xi_{t}$, but they do respond contemporaneously to a shock to the investment-savings relationship, $z_{t}$. These restrictions are required for us to be able to identify the structural shocks of interest in the VAR model based on shortrun identifying restrictions. Given this informational constraint, household and firms form expectations based on the information set $\mathcal{I}_{t-1}$.

Given the computational cost of evaluating higher-dimensional models, we focus on the estimation of one parameter only in the simulation study. For expository purposes, we 
concentrate on the problem of estimating the probability of not adjusting prices, $\alpha$, by matching the impulse responses of inflation and of the interest rate with the remaining parameters set to their population values in estimation. The population parameters in the data generating process are $\sigma=1, \alpha=0.75, \beta=0.99, \phi_{\pi}=1.5, \phi_{x}=0.125, \omega=1$, $\rho_{r}=0.75, \rho_{z}=0.90, \theta=6, \sigma^{z}=0.30, \sigma^{r}=0.20$. It can be shown that the parameter $\alpha$ is strongly identified.

This DSGE model may equivalently be expressed in the state-space representation

$$
\begin{aligned}
& x_{t}=A x_{t-1}+B \varepsilon_{t}, \\
& y_{t}=C x_{t},
\end{aligned}
$$

where $x_{t}$ is a vector of state variables, $\varepsilon_{t}$ is a vector that consists of the technology shock and the monetary policy shock, and $y_{t}$ is a vector that consists of inflation and the interest rate. $A, B$ and $C$ are matrices of suitable dimensions. Substitution of $x_{t}$ in equation (33) yields the structural moving average representation of $y_{t}$ in terms of current and lagged structural shocks $\varepsilon_{t}$. This moving average representation is invertible because, given our population parameter values, the eigenvalues of $A$ are strictly less than unity in modulus, so Fernandez-Villaverde, Rubio-Ramirez, Sargent and Watson's (2007) condition for the existence of an infinite-order VAR representation is satisfied. This structural $\operatorname{VAR}(\infty)$ model for $y_{t}$ in turn may be approximated by a finite-order structural VAR model (see Inoue and Kilian 2002). Because the structural impact multiplier matrix of the VAR model, $C B$, is lower triangular given the informational constraints discussed earlier, we can recover the two structural shocks $\varepsilon_{t}^{z}$ and $\varepsilon_{t}^{r}$ by applying a lower triangular Cholesky decomposition to the residual covariance matrix with the diagonals of the decomposition normalized to be positive.

It may be tempting to match the structural VAR impulse response estimates with the value of $\gamma(\alpha)$ implied by the underlying DSGE model, which has a $\operatorname{VAR}(\infty)$ representation. This approach is problematic because it creates a mismatch with the structural impulse 
responses, which are estimated based on a finite-order approximation to this $\operatorname{VAR}(\infty)$ model. In practice, we therefore derive the population value of $\gamma(\alpha)$ based on the finiteorder VAR representation of the macroeconomic model with the same choice of $p$ as in the empirical VAR model, denoted $\gamma_{p}(\alpha)$. Let $\Gamma_{j}=E\left(y_{t} y_{t-j}^{\prime}\right)$ denote the population autocovariances implied by the state space representation given a structural parameter value. Then the population parameter values of the $\operatorname{VAR}(p)$ model fitted to data generated by the model may be expressed as:

$$
\begin{aligned}
\underset{2 p \times 2}{\mathbf{\Phi}}= & {\left[\begin{array}{cccc}
\Gamma_{0} & \Gamma_{1} & \cdots & \Gamma_{p-1} \\
\Gamma_{1}^{\prime} & \Gamma_{0} & \cdots & \Gamma_{p-2} \\
\vdots & \vdots & \ddots & \vdots \\
\Gamma_{p-1}^{\prime} & \Gamma_{p-2}^{\prime} & \cdots & \Gamma_{0}
\end{array}\right]^{-1}\left[\begin{array}{c}
\Gamma_{1}^{\prime} \\
\Gamma_{2}^{\prime} \\
\vdots \\
\Gamma_{p}^{\prime}
\end{array}\right], } \\
\underset{2 \times 2}{\mathbf{\Sigma}}= & \Gamma_{0}-\left[\begin{array}{llll}
\Gamma_{1} & \Gamma_{2} & \cdots & \Gamma_{p}
\end{array}\right]\left[\begin{array}{cccc}
\Gamma_{0} & \Gamma_{1} & \cdots & \Gamma_{p-1} \\
\Gamma_{1}^{\prime} & \Gamma_{0} & \cdots & \Gamma_{p-2} \\
\vdots & \vdots & \ddots & \vdots \\
\Gamma_{p-1}^{\prime} & \Gamma_{p-2}^{\prime} & \cdots & \Gamma_{0}
\end{array}\right]\left[\begin{array}{c}
\Gamma_{1}^{\prime} \\
\Gamma_{2}^{\prime} \\
\vdots \\
\Gamma_{p}^{\prime}
\end{array}\right]
\end{aligned}
$$

The population structural impulse responses can be calculated from the slope coefficients $\Phi$ and the reduced-form error covariance matrix $\boldsymbol{\Sigma}$.

The Monte Carlo study consists of the following steps:

1. First, we generate 500 synthetic data sets of length $T$ for inflation and the interest rate from the New Keynesian model evaluated at the true parameter values. We focus on two alternative sample sizes: $T=100$ and $T=232$. The shorter sample corresponds to the length of a quarterly time series starting in 1984 with the onset of the Great Moderation. The longer sample corresponds to the period from 1950 to 2008, which represents another common situation in the empirical literature.

2. For each synthetic data set, we fit a bivariate $\operatorname{VAR}(p)$ model for inflation and the 
interest rate and estimate the four structural impulse response functions at horizons $0, \ldots, H$. All freely estimated elements of these impulse response functions are stacked into a vector and denoted by $\widehat{\gamma}_{T}$. To this end, we impose that inflation does not react contemporaneously to the second structural shock in the VAR, which identifies this shock as the monetary policy shock. Using the standard residual-based bootstrap for VAR models, we bootstrap the $\operatorname{VAR}(p)$ model and estimate a vector of bootstrap structural impulse responses $\widehat{\gamma}_{T}^{*(j)}$ for $j=1, \ldots, B$, where $B=500$. For each of the $B$ bootstrapped VAR estimates, $\widehat{\gamma}_{T}^{*(j)}$, the residual-based bootstrap is applied and $B$ vectors of bootstrap structural impulse responses, $\widehat{\gamma}_{T}^{* *(j, k)}$, are computed for $k=1,2, \ldots, B$.

3. We estimate $\alpha$, treating the other parameters as known in the estimation for computational simplicity. Define $\widehat{\alpha}_{T}$ by

$$
\begin{aligned}
\widehat{\alpha}_{\text {opt }, T} & =\operatorname{argmin}_{\alpha \in A}\left(\widehat{\gamma}_{T}-\gamma_{p}(\alpha)\right)^{\prime} W_{\text {opt }, T}\left(\widehat{\gamma}_{T}-\gamma_{p}(\alpha)\right), \\
\widehat{\alpha}_{\text {diag }, T} & =\operatorname{argmin}_{\alpha \in A}\left(\widehat{\gamma}_{T}-\gamma_{p}(\alpha)\right)^{\prime} W_{\text {diag }, T}\left(\widehat{\gamma}_{T}-\gamma_{p}(\alpha)\right),
\end{aligned}
$$

where $W_{\text {opt }, T}=\left[(1 / B) \sum_{j=1}^{B}\left(\widehat{\gamma}_{T}^{*(j)}-\overline{\widehat{\gamma}}_{T}^{*}\right)\left(\widehat{\gamma}_{T}^{*(j)}-\overline{\widehat{\gamma}}_{T}^{*}\right)^{\prime}\right]^{-1}$ and $W_{\text {diag }, T}$ is the diagonal matrix whose diagonal elements are given by the reciprocal of the diagonal elements of $(1 / B) \sum_{j=1}^{B}\left(\widehat{\gamma}_{T}^{*(j)}-\bar{\gamma}_{T}^{*}\right)\left(\widehat{\gamma}_{T}^{*(j)}-\bar{\gamma}_{T}^{*}\right)^{\prime}$

4. Let $\widehat{\alpha}_{T}^{*}$ be the bootstrap analogue of $\widehat{\alpha}_{T}$. Then

$$
\begin{aligned}
& \widehat{\alpha}_{o p t, T}^{*(j)}=\operatorname{argmin}_{\alpha \in A}\left(\widehat{\gamma}_{T}^{*(j)}-\gamma_{p}(\alpha)-\widehat{\gamma}_{T}+\gamma_{p}\left(\widehat{\alpha}_{o p t, T}\right)\right)^{\prime} W_{o p t, T}^{*(j)}\left(\widehat{\gamma}_{T}^{*(j)}-\gamma_{p}(\alpha)-\widehat{\gamma}_{T}+\gamma_{p}\left(\widehat{\alpha}_{o p t, T}\right)\right), \\
& \widehat{\alpha}_{\text {diag }, T}^{*(j)}=\operatorname{argmin}_{\alpha \in A}\left(\widehat{\gamma}_{T}^{*(j)}-\gamma_{p}(\alpha)-\widehat{\gamma}_{T}+\gamma_{p}\left(\widehat{\alpha}_{\text {diag }, T}\right)\right)^{\prime} W_{\text {diag }, T}^{*(j)}\left(\left(_{\gamma_{T}^{*(j)}}^{*(j)}-\gamma_{p}(\alpha)-\widehat{\gamma}_{T}+\gamma_{p}\left(\widehat{\alpha}_{\text {diag }, T}\right)\right),\right.
\end{aligned}
$$

where $W_{o p t, T}^{*(j)}=\left[(1 / B) \sum_{k=1}^{B}\left(\widehat{\gamma}_{T}^{* *(j, k)}-\bar{\gamma}_{T}^{* *(j)}\right)\left(\widehat{\gamma}_{T}^{* *(j, k)}-\bar{\gamma}_{T}^{* *(j)}\right)^{\prime}\right]^{-1}$ and $W_{\text {diag, } T}^{*(j)}$ is the diagonal matrix whose diagonal elements are given by the reciprocal of the diagonal 
elements of $(1 / B) \sum_{k=1}^{B}\left(\widehat{\gamma}_{T}^{* *(j, k)}-\bar{\gamma}_{T}^{* *(j)}\right)\left(\widehat{\gamma}_{T}^{* *(j, k)}-\bar{\gamma}_{T}^{* *(j)}\right)^{\prime}$. Using these bootstrap estimates we construct nominal 90\% confidence intervals based on Hall's (1992) percentile interval to allow for the fact that the asymptotic distribution may not be normal, depending on the choice of the weighting matrix. The coverage rates are evaluated based on the relative frequency with which these interval estimators include $\alpha$.

Our simulation evidence is necessarily tentative, but nevertheless provides some useful insights. Table 1 summarizes the properties of the point estimator of $\alpha$. Because the quality of the VAR approximation depends on the lag order $p$, we report results for a range of $p$. We also consider a range of values for $H$ to allow for situations in which the asymptotic theory developed on this paper applies $(H>p)$ as well as for situations in which the conventional asymptotic theory for impulse response matching estimators applies $(p \leq H)$. The upper panel of Table 1 shows that the estimator based on the optimal weighting matrix tends to have lower bias, a lower standard deviation and a lower root MSE (RMSE) than the estimator based on the diagonal weighting matrix.

The left panel of Table 2 reports the effective coverage probabilities of the corresponding nominal $90 \%$ bootstrap confidence intervals for $\alpha$. The confidence intervals based on the diagonal weighting matrix tend to be more robust to the choice of $p$ and $H$ than the confidence intervals based on the optimal weighting matrix. The latter interval lacks coverage accuracy for large $H$ and small $T$. Even the coverage rates of the interval based on the diagonal matrix are too low for $T=100$. Generally, its coverage rates are reasonably close to their nominal levels only for $T=232$. The coverage deficiencies in small samples can be traced to approximation error in the VAR representation of the DSGE model. When bootstrapping the state-space representation of the DSGE model directly rather than its VAR approximation, as shown in the right panel of Table 2, high coverage accuracy is obtained even for $T=100$, albeit at the cost of taking a stand on the parametric structure of the data generating process. The best results for the DSGE model bootstrap are obtained based on the optimal weighting matrix. 
When doubling the post-war sample size for expository purposes, as shown in the bottom panel of Table 2, the differences in coverage accuracy between the VAR model bootstrap and the DSGE model bootstrap tends to vanish. Table 2 shows that the VAR bootstrap based on the diagonal weighting matrix delivers reasonably accurate intervals for $T=464$, as predicted by asymptotic theory. The coverage accuracy of the VAR bootstrap based on the optimal weighting matrix also improves substantially, but remains systematically lower than for the VAR bootstrap based on the diagonal weighting matrix. We conclude that the use of the diagonal weighting matrix is clearly preferred for inference.

The baseline simulation results in Table 2 pertain to strongly identified structural DSGE model parameters. Table 3 provides additional simulation evidence for VAR-based bootstrap confidence intervals that allow for weak identification. They tend to be reasonably accurate even for $T=100$ and compare well to the their DSGE-model-based bootstrap counterparts. We conclude that the accurate inference allowing for weak identfication is possible even for realistic sample sizes.

\section{Empirical Application}

For the empirical application, we consider a prototypical medium-scale New Keynesian DSGE model (see, e.g., Christiano, Eichenbaum, and Evans 2005; Smets and Wouters 2007; Altig, Christiano, Eichenbaum and Lindé 2011; Guerron-Quintana, Inoue, and Kilian 2013). Since this class of models has been extensively discussed in the macroeconomics literature, we provide only a brief summary. The main features of the model are as follows: The economy grows along a stochastic path; prices and wages are assumed to be sticky à la Calvo; preferences display internal habit formation; investment is costly; and finally, there are three sources of uncertainty: neutral and capital embodied technology shocks, and monetary shocks. 


\subsection{Households}

The economy is populated by a continuum of households. Every period households must decide how much to consume, work, and invest. In addition, they must choose the amount of government bonds. Agents in the economy have access to complete markets; such an assumption is needed to eliminate wealth differentials arising from wage heterogeneity. Households maximize the expected present discounted value of utility

$$
\mathbb{E}_{0} \sum_{t=0}^{\infty} \beta^{t}\left[\log \left(C_{t}-b C_{t-1}\right)-A \int_{0}^{1} \frac{h_{j, t}^{1+v}}{1+v} d j\right]
$$

subject to

$$
P_{t} C_{t}+P_{t}\left(I_{t}+a\left(u_{t}\right) \bar{K}_{t}\right) / \Psi_{t}+B_{t+1}=R_{t}^{K} u_{t} \bar{K}_{t}+\int_{0}^{1} W_{j, t} h_{j, t} d j+R_{t-1} B_{t}+\Pi_{t}+T_{t}
$$

and

$$
\bar{K}_{t+1}=(1-\delta) \bar{K}_{t}+I_{t}\left(1-S\left(\frac{I_{t}}{I_{t-1}}\right)\right)
$$

Here, $\mathbb{E}_{t}$ is the time $t$ expectation operator conditional on the information set of the household; preferences display internal habit formation measured by $b \in(0,1)$; and $S($.$) is a$ function reflecting the costs associated with adjusting the investment portfolio. This function is assumed to be increasing and convex satisfying $S=S=0$ and $S^{\prime \prime}>0$ in the steady state. $T_{t}$ corresponds to lump-sum transfers from the government to the household. $B_{t}$ is the individual demand for one-period government bonds, which pay the gross nominal interest rate $R_{t}$. As in the related literature, it is assumed that physical capital can be used with different intensities (see, e.g., Christiano, Eichenbaum, and Evans; 2005). Using capital with intensity $u_{t}$ yields the return $R_{t}^{K} u_{t} \bar{K}_{t}$ but entails a cost $a\left(u_{t}\right)$, which satisfies $a(1)=0 ; a^{\prime \prime}(1)>0 ; a^{\prime}(1)>0$. For future reference, we define $\sigma_{a}=a^{\prime \prime}(1) / a^{\prime}(1)$. Finally, $\Pi_{t}$ corresponds to profits from producers. $\Psi_{t}$ is an investment-specific disturbance, which, 
following the literature, is assumed to grow at rate $\mu_{\Psi, t}=\log \left(\Psi_{t} / \Psi_{t-1}\right)$, where

$$
\mu_{\Psi, t}=\left(1-\rho_{\psi}\right) \mu_{\psi}+\rho_{\mu, \psi} \mu_{\Psi, t-1}+\sigma_{\epsilon, \psi} \epsilon_{\Psi, t},
$$

and $\epsilon_{\Psi, t}$ is distributed $\mathcal{N} \mathcal{I D}(0,1)$.

\subsection{Wage Setting}

Households sell differentiated labor services $h_{j, t}$ to a competitive firm that aggregates labor and sells it to final firms. This labor aggregator pays $W_{j, t}$ for each unit of differentiated labor of type $j$. The technology used by the aggregator is

$$
H_{t}=\left[\int_{0}^{1} h_{j, t}^{1 / \lambda_{w}} d j\right]^{\lambda_{w}}, \quad 1<\lambda_{w} .
$$

It is straightforward to show that the relationship between the labor aggregate, $H_{t}$, and the aggregate wage, $W_{t}$, is given by

$$
h_{j, t}=\left[\frac{W_{j, t}}{W_{t}}\right]^{-\lambda_{w} /\left(\lambda_{w}-1\right)} H_{t} .
$$

To induce wage sluggishness, it is assumed that there exists a labor union representing all workers of type $j$. Each period, the union sets wages in a Calvo fashion. In particular, with exogenous probability $\xi_{w}$ a union does not re-optimize wages each period. In that case, wages are set according to the rule of thumb $W_{j, t}=\pi^{1-\iota_{w}}\left(\pi_{t}\right)^{\iota w} W_{j, t-1} \mu_{z^{+}}$. Here, $\mu_{z^{+}}$ is the average growth rate of the economy, as defined below, and $\iota_{w}$ is the degree of wage indexation to inflation.

\subsection{Firms}

There is a continuum of monopolistically competitive firms indexed by $i \in[0,1]$, each producing an intermediate good from capital services, $k_{i, t}$, and labor services, $H_{i, t}$. Firms 
rent capital and labor in perfectly competitive factor markets. The production function is given by

$$
Y_{i, t}=k_{i, t}^{\alpha}\left(z_{t} H_{i, t}\right)^{1-\alpha}-z_{t}^{+} \psi
$$

where $\psi$ is a fixed cost of production. The technology shock, $z_{t}$, grows at rate $\mu_{z, t}=$ $\log \left(z_{t} / z_{t-1}\right)$, which is assumed to follow the process

$$
\mu_{z, t}=\mu_{z}+\sigma_{\epsilon, \mu} \epsilon_{z, t}
$$

where $\epsilon_{z, t}$ is distributed $\mathcal{N} \mathcal{I D}(0,1)$. The aggregate trend $z_{t}^{+}=\Psi_{t}^{\alpha /(1-\alpha)} z_{t}$ grows at rate $\mu_{z^{+}, t^{2}}$

Intermediate firms must borrow the wage bill in advance. As a consequence, the cost of hiring one unit of labor is $W_{t} R_{t}$. These firms choose prices to maximize the present value of profits; prices are set in Calvo fashion; i.e., each period, firms optimally revise their prices with an exogenous probability $1-\xi_{p}$. If, instead, a firm does not re-optimize its price, then the price is updated according to the rule: $P_{i, t}=\pi P_{i, t-1}$. Here, $\pi$ is steady-state inflation.

There is a competitive firm that produces the final good using intermediate goods according to the technology

$$
Y_{t}=\left[\int_{0}^{1} Y_{j, t}^{1 / \lambda_{p}} d j\right]^{\lambda_{p}}
$$

The parameter $\lambda_{p}$ determines the degree of monopoly power enjoyed by intermediate producers.

\subsection{Government}

As in most of the recent New Keynesian literature, we assume a cashless economy (see Woodford 2003). The monetary authority sets the short-term interest rate according to a Taylor rule. In particular, the central bank smoothes interest rates and responds to

\footnotetext{
${ }^{2}$ The growth term in the fixed cost is needed for a well-defined steady state to exist about which the model can be solved.
} 
deviations of actual inflation from steady-state inflation, $\pi$, and deviations of output from its target level, $\mathcal{Y}$.

$$
\frac{R_{t}}{R}=\left(\frac{R_{t-1}}{R}\right)^{\rho_{r}}\left[\left(\frac{\pi_{t}}{\pi}\right)^{\phi_{\pi}}\left(\frac{\mathcal{Y}_{t}}{\mathcal{Y}}\right)^{\phi_{y}}\right]^{1-\rho_{r}} \exp \left(\sigma_{\epsilon, r} \epsilon_{r, t}\right) .
$$

The term $\epsilon_{r, t}$ is a random shock to the systematic component of monetary policy and is assumed to be standard normal; $\sigma_{\epsilon, r}$ is the standard deviation of the monetary shock. Following Christiano, Trabandt and Walentin (2011), $\mathcal{Y}_{t}$ corresponds to de-trended GDP

such that $\mathcal{Y}_{t}=\frac{C_{t}+I_{t} / \Psi_{t}+G_{t}}{z_{t}^{+}} . R$ is the steady-state gross nominal interest rate. Finally, we assume that government spending is given by $G_{t}=g z_{t}^{+}$. Here, $g$ is a constant and the government uses lump-sum taxes to finance its purchases.

\subsection{Estimation}

We estimate the model in two stages. First, a stationary $\operatorname{VAR}(2)$ model is used to recover the dynamic responses of the model variables to three structural innovations: the monetary policy, the shock to the growth of neutral productivity, and the shock to the growth rate of investment-specific technology. The sample extends from 1951Q1 to 2008Q4. The set of VAR variables include the growth rate of the relative price of investment, the growth rate of the real GDP-to-hours ratio, inflation, the unemployment rate, capacity utilization, the $\log$ of hours, the log of real GDP-to-hours ratio minus the log of real wages, the log of the nominal consumption-to-nominal GDP ratio, the log of the nominal investment-to-nominal GDP ratio, vacancies, the job separation rate, the job finding rate, the log of hours-to-labor force ratio, and the fed funds rate. ${ }^{3}$

Second, the structural parameters in the model are estimated by minimizing the distance between the DSGE model's impulse responses to the structural shocks and the corresponding structural VAR responses. There are three identifying assumptions imposed on

\footnotetext{
${ }^{3}$ For additional details on the estimation of the structural VAR, the reader may consult Altig et al. (2011) and Christiano et al. (2011). Note that Christiano et al. (2011) treat the federal funds rate as I(0) in their analysis.
} 
the structural VAR model. First, the only variable that the monetary policy shock affects contemporaneously is the federal funds rate. Second, the only shocks that affect labor productivity in the long run are the two technology shocks. Third, the only shock that affects the price of investment relative to consumption in the long run is the innovation to the investment-specific shock. All these identifying assumptions are satisfied in the underlying DSGE model as well. We follow the literature in matching the responses of nine of the fourteen model variables to each of the identified structural shocks.

As is standard in the literature, in estimating $\theta$ a subset of the structural parameters is set to values typically imposed in the literature and treated as known: The capital share is $\alpha=0.25$; the depreciation rate is $\delta=0.025$; the discount factor is $\beta=0.999$; the steadystate gross inflation is $\pi=1.0083$; the government consumption to GDP ratio is 0.2 ; the relative price of capital in steady state is 1 ; the wage indexation parameter is $\iota_{w}=1$; the wage markup is $\lambda_{w}=1.01$; wage stickiness $\xi_{w}=0.75$; the gross neutral technology growth is $\mu_{z}=1.0041 ;$ and the gross investment technology growth is $\mu_{\Psi}=1.0018$.

We consider two alternative estimation methods. The first method corresponds to the frequentist impulse matching approach as outlined in Rotemberg and Woodford (1997) and Christiano, Eichenbaum, and Evans (2005). The second method corresponds to the Bayesian impulse matching framework recently proposed by Christiano, Trabandt and Walentin (2011). Both methods are based on the diagonal weighting matrix.

\subsubsection{Results}

Table 4 presents the estimates of the structural parameters based on alternative choices of the maximum horizon of the impulse response functions. We evaluate the structural impulse responses at horizons $0,1, \ldots, H$ with $H \in\{15,19\}$. The order condition $q \leq k$, where $q$ is the number of impulse response parameters to be matched and $k$ is the number of VAR model parameters, is violated for $H=19$, given that the approximating VAR model in this example includes 14 variables and 2 lags. The order condition is satisfied for 
$H=15$.

The column labelled Frequentist reports the results from the impulse response matching estimator based on the diagonal weighting matrix that performed well in the simulation study. The first column corresponds to the point estimate for each structural parameter and the second column shows the standard error. Given the asymptotic normality of this estimator, it makes sense to base inference on these two summary statistics. Alternatively, one could have reported bootstrap percentile intervals. We focus on the standard errors to conserve space.

The next three columns labelled Bayesian report results obtained from the quasiposterior distribution. The first column shows the mode, median and mean of the quasiposterior, respectively, as three alternative point estimates of the structural parameters, and the second column provides the corresponding standard errors. All Bayesian results are based on the diagonal weighting matrix and rely on the sandwich formula of Chernozhukov and Hong (2003).

Finally, the column $C T W$ reports the mean and standard deviation of the quasiposterior distributions, computed as in Christiano, Trabandt, and Walentin (2011). As we showed earlier, the variance of the quasi-posterior cannot be used to estimate the asymptotic variance of the structural parameters, but these standard deviations are reported for comparison.

The qualitative pattern of the results is similar for all choices of $H$. Table 4 shows that the point estimates are quite robust across alternative estimation methods. This result is not surprising, because all point estimates reported in these tables are consistent. In contrast, approximating the asymptotic standard errors of the parameter estimates based on the standard deviation of the quasi-posterior as in CTW results in much lower standard error estimates than using methods that are asymptotically valid. The latter estimates are higher by a factor of 3 in many cases. For example, the standard error of the price markup for $H=15$ increases from 0.08 to 0.22 . Whereas the confidence interval obtained 
by adding \pm 1.96 standard deviations of the quasi-posterior to the point estimate reported in the $C T W$ column does reject the null hypothesis that there is no markup (i.e., $\lambda_{p}=1$ ), 95\% confidence intervals based on the point estimates and standard errors in the columns labelled Frequentist and Bayesian do not. Likewise, the standard error of the consumption habit parameter, $b$, increases threefold compared with the asymptotically invalid estimate reported in the $C T W$ column. For $H=15$, it increases from 0.02 to 0.07 . Similarly, the policy reaction function parameters are estimated very imprecisely. For example, $\phi_{\pi}$ is no longer statistically significantly different from zero when conducting inference based on the Bayesian intervals, although it does remain significantly different from zero using the Frequentist interval. The fact that Bayesian priors affect the results is not unexpected, given the high dimensionality of the model. For $H=19$, broadly similar results are obtained.

A parameter of particular interest is the price stickiness parameter. The length of the price contracts is defined as $1 /\left(1-\xi_{p}\right)$ quarters, where $\xi_{p}$ is the probability of not reoptimizing prices today. There is an active literature on measuring the degree of price rigidity at the micro level (see, e.g., Klenow and Kryvtsov (2008), Nakamura and Steinsson (2008)). For example, Klenow and Kryvtsov (2008) provide evidence that price contracts last, on average, about 2.3 quarters. Based on the point estimates for $H=15$ in Table 4 , a researcher would have concluded that the length of a price contract is $2.94,2.65$ or 2.64, respectively. The $95 \%$ confidence interval constructed from the information in the $C T W$ column ranges from 2.23 to 3.25 and includes the value of 2.3 from the micro literature. The 95\% confidence interval for the length of the price contract implied by the Frequentist estimates ranges from 2.12 to 4.79 and includes that value as well, as do the corresponding three $95 \%$ confidence intervals implied by the entries in the Bayesian columns of Table 3.

This pattern of results changes for $H=19$. In the latter case, $q>k$, and the conventional asymptotic theory for the CMD estimator breaks down. The results in the CTW column of Table 4 imply a $95 \%$ confidence interval with a lower bound of 2.67 that excludes 2.3 for $H=19$, whereas the three asymptotically valid intervals computed based on the en- 
tries in the Bayesian columns all include the value of 2.3, suggesting that the DSGE model estimate is consistent with the micro evidence. These estimates are all based on the same prior and hence directly comparable. This example illustrates that the choice of estimation method may affect one's views of whether the macroeconomic evidence is compatible with the length of the price spells found in the micro literature. In contrast, the corresponding 95\% confidence interval based on the Frequentist method does not include 2.3 for $H=19$, illustrating the influence of the prior on the estimates.

The results in Table 4 are based on the conventional premise in empirical work that the structural parameters of interest are strongly identified. The sensitivity of the results to the prior is an indication that the structural parameters may not be strongly identified. Table 5 presents an alternative set of results that allows for the possibility that at least some parameters are only weakly identified. These results are of particular interest for applied work, as none of the currently available methods of inference allows for weak identification of the structural parameters, which is a common problem in the estimation of DSGE models in practice, including the type of DSGE model considered here. We again focus on $H=15$ and $H=19$, for expository purposes. Of particular interest is a comparison with the Frequentist results in Table 4.

Table 5 shows the lower and upper endpoints of the pointwise $95 \%$ interval for each parameter. Allowing for weak identification can affect the substantive conclusions. For example, for both choices of $H$ the null hypothesis that there is no markup (i.e., $\lambda_{p}=1$ ) is rejected. This result differs from the Frequentist results in Table 4, which did not allow us to reject this null hypothesis for either $H$. On the other hand, the results for the length of the price contract are qualitatively consistent with Frequentist results in Table 4 that were obtained under the premise of strong identification. Whereas the confidence interval for $H=15$ in Table 5 includes 2.3 quarters, the confidence interval for $H=19$ in Table 5 does not. 


\section{Concluding Remarks}

One of the leading methods of estimating the structural parameters of DSGE models is the VAR-based impulse response matching estimator. The existing asymptotic theory for this estimator does not cover situations in which the number of impulse response parameters $(q)$ exceeds the number of VAR model parameters $(k)$. Such situations often arise in applied work. We established the consistency of the impulse response matching estimator in this situation, derived its asymptotic distribution and showed how this distribution can be approximated by bootstrap methods that remain asymptotically valid whether $q>k$ or $q \leq k$. Our results provide formal guidance on how to conduct inference about structural parameters in DSGE models. We also discussed implications of these results for tests of overidentifying restrictions.

Our analysis sheds new light on the choice of the weighting matrix. On the one hand, we showed that the impulse response matching estimator based on the optimal weighting matrix, while remaining consistent under our assumptions, does not have an asymptotic normal distribution. Approximating its distribution requires suitable bootstrap methods in practice. On the other hand, we provided a formal justification for the use of bootstrap methods in conducting inference about impulse response matching estimators based on the diagonal weighting matrix. The distribution of the latter estimator was shown to be asymptotically normal. This result is important because this estimator to date has been used without a formal asymptotic justification having been provided for the practically relevant case of $q>k$. Our analysis also showed that special care is required to ensure that Bayesian methods of inference remain valid from an asymptotic point of view.

We compared the finite-sample accuracy of impulse response matching estimators based on alternative weighting matrices by simulation. A Monte Carlo study based on a smallscale New Keynesian macroeconomic model suggested that the proposed bootstrap interval estimators based on the diagonal weighting matrix are more accurate in finite samples than estimators based on the optimal weighting matrix and more robust to the choice of the lag 
order of the approximating VAR model and the horizon. The coverage accuracy in small samples may be improved by bootstrapping the state-space representation of the DSGE model instead of its VAR representation, albeit at the cost of imposing more parametric structure on the data generating process. For larger samples, these two approaches tend to have similar coverage accuracy.

Finally, we extended the analysis to cover weakly identified DSGE model parameters. Weak identification is a pervasive problem in medium-scale DSGE models. Although several solutions to this problem have been proposed in the recent literature, none apply to impulse response matching estimators. Thus, our analysis greatly extends the range of applications of the impulse response matching estimator. We showed that robustness to weak identification may be achieved by inverting the Wald test statistic of the structural impulse responses to form a joint confidence set and by applying the projection method to recover confidence intervals for individual structural parameters. The proposed method remains asymptotically valid whether $q>k$ or $q \leq k$. In a simulation study it performed well even in realistically small samples.

We illustrated the use of the various new methods proposed in this paper in practice. When estimating a prototypical medium-scale New Keynesian DSGE model based on Christiano, Trabandt and Walentin (2011), inference based on the alternative methods proposed in this paper generated substantively different conclusions than methods based on the standard deviation of the quasi-posterior distribution of the structural parameters. For example, whereas the latter method suggested that the macro evidence is inconsistent with micro evidence on the degree of price stickiness at conventional significance levels, given a maximum horizon of 19 quarters, we demonstrated that this result is overturned when using asymptotically valid Bayesian methods of inference. Substantively different results regarding the degree of price stickiness were obtained using frequentist methods, which suggested that the macroeconomic estimates based on a maximum horizon of 19 quarters are inconsistent with the micro evidence. The latter result was shown to be robust to 
allowing for weak identification.

An interesting topic for future research would be to extend the impulse response matching estimator to nonlinear DSGE models such as the Markov-switching DSGE model in Liu et al. (2011). As Liu et al. (2011) emphasize, the standard approach to analyzing business cycle fluctuations is based on medium-scale constant parameter DSGE models such as the class of models considered in our analysis. DSGE models that allow for Markov switching in the volatility or in other model features, in contrast, do not have a constant-coefficient linear representation. Such models are not covered by our analysis. In fact, there are no results on impulse response matching estimators for Markov switching models even when $q \leq k$. There are several challenges. First, it remains to be shown whether models of this type have a Markov switching VAR (MS-VAR) representation. For constant-coefficient DSGE models, Fernandez-Villaverde, Rubio-Ramirez, Sargent and Watson (2007) provide specific conditions that allow researchers to verify the existence of a linear VAR representation of the DSGE model. To our knowledge, no such representation theorem exists for Markov switching DSGE models. Second, even if we take the existence of an MS-VAR representation for granted, impulse responses in nonlinear models have no closed-form solutions. They depend on the history of the data and the magnitude of the shocks and must be computed by Monte Carlo integration. The absence of a closed-form solution makes it difficult to apply the analytic tools that our current paper utilizes. Third, even assuming that one integrates out the histories in the MS-DSGE model and its MS-VAR approximation, the impulse responses are not uniquely defined. One possible solution would be to appropriately weight the responses obtained for different magnitudes of shocks. 


\section{Appendix A: Proofs}

Proof of Theorem 1. It follows from the definitions of $S_{j}$ and Assumptions (b) and (c) that

$$
\begin{aligned}
& \Upsilon_{T} S^{\prime}\left(\gamma\left(\bar{X}_{T}\right)-f(\theta)\right)=\left[\begin{array}{c}
S_{0}^{\prime} B_{0} Z_{T}+T^{-\frac{1}{2}} S_{0}^{\prime} B_{1}\left(Z_{T} \otimes \times Z_{T}\right)+\cdots+T^{-\frac{H}{2}} S_{0}^{\prime} B_{H}\left(Z_{T} \otimes \cdots \otimes Z_{T}\right)+o_{p}\left(T^{-\frac{H}{2}}\right) \\
T^{\frac{j_{1}}{2}}\left(S_{1}^{\prime} B_{0} Z_{T}+T^{-\frac{1}{2}} S_{1}^{\prime} B_{1}\left(Z_{T} \otimes Z_{T}\right)+\cdots+T^{-\frac{H}{2}} S_{1}^{\prime} B_{H}\left(Z_{T} \otimes \cdots \otimes Z_{T}\right)+o_{p}\left(T^{-\frac{H}{2}}\right)\right) \\
\vdots \\
T^{\frac{j_{r}}{2}}\left(S_{r}^{\prime} B_{0} Z_{T}+T^{-\frac{1}{2}} S_{r}^{\prime} B_{1}\left(Z_{T} \otimes Z_{T}\right)+\cdots+T^{-\frac{H}{2}} S_{r}^{\prime} B_{H}\left(Z_{T} \otimes \cdots \otimes Z_{T}\right)+o_{p}\left(T^{-\frac{H}{2}}\right)\right)
\end{array}\right] \\
& +\left[\begin{array}{c}
T^{\frac{1}{2}} S_{0}^{\prime}\left(f\left(\theta_{0}\right)-f(\theta)\right) \\
T^{\frac{j_{1}+1}{2}} S_{1}^{\prime}\left(f\left(\theta_{0}\right)-f(\theta)\right) \\
\vdots \\
T^{\frac{j_{r}+1}{2}} S_{r}^{\prime}\left(f\left(\theta_{0}\right)-f(\theta)\right)
\end{array}\right] \\
& =\left[\begin{array}{c}
S_{0}^{\prime} B_{0} Z_{T} \\
S_{1}^{\prime} B_{j_{1}}\left(Z_{T} \otimes \cdots \otimes Z_{T}\right) \\
\vdots \\
S_{r}^{\prime} B_{j_{r}}\left(Z_{T} \otimes \cdots \otimes Z_{T}\right)
\end{array}\right]+\left[\begin{array}{c}
T^{\frac{1}{2}} S_{0}^{\prime}\left(f\left(\theta_{0}\right)-f(\theta)\right) \\
T^{\frac{j_{1}+1}{2}} S_{1}^{\prime}\left(f\left(\theta_{0}\right)-f(\theta)\right) \\
\vdots \\
T^{\frac{j_{r}+1}{2}} S_{r}^{\prime}\left(f\left(\theta_{0}\right)-f(\theta)\right)
\end{array}\right]+o_{p}(1)
\end{aligned}
$$

where $o_{p}(1)$ is uniform in $\theta \in \Theta$ due to the continuity of $f(\cdot)$ and the compactness of $\Theta$. Because of Assumptions (a)-(d) and because of the continuity of eigenvalues as a function of matrices, we have

$$
\Upsilon_{T} S^{\prime}\left(\gamma\left(\bar{X}_{T}^{*}\right)-\gamma\left(\bar{X}_{T}\right)\right) \stackrel{d}{\rightarrow}\left[\begin{array}{c}
S_{0}^{\prime} B_{0} Z^{*} \\
S_{1}^{\prime} B_{j_{1}}\left(Z^{*} \otimes \cdots \otimes Z^{*}\right) \\
\vdots \\
S_{r}^{\prime} B_{j_{r}}\left(Z^{*} \otimes \cdots \otimes Z^{*}\right)
\end{array}\right]=\xi^{*} \text { in prob- } P .
$$

It follows from (43) and Assumptions (a)-(e) that

$$
\Upsilon_{T} S^{\prime} \widehat{\Sigma}_{T}^{*} S \Upsilon_{T}=E^{*}\left(\xi^{*} \xi^{* \prime}\right)+o_{p}^{*}(1)=J+o_{p}^{*}(1) \text { in prob- } P
$$


It follows from (42) and (44) that the objective function for $\widehat{\theta}_{o p t, T}$ is asymptotically proportional to

$$
\begin{aligned}
& \frac{1}{T^{j_{r}+1}}\left(\widehat{\gamma}_{T}-f(\theta)\right)^{\prime} \widehat{\Sigma}_{T}^{*-1}\left(\widehat{\gamma}_{T}-f(\theta)\right) \\
= & \frac{1}{T^{j_{r}+1}}\left(\widehat{\gamma}_{T}-f(\theta)\right)^{\prime} S \Upsilon_{T}\left(\Upsilon_{T} S^{\prime} \widehat{\Sigma}_{T}^{*} S \Upsilon_{T}\right)^{-1} \Upsilon_{T} S^{\prime}\left(\widehat{\gamma}_{T}-f(\theta)\right) \\
= & \left(f\left(\theta_{0}\right)-f(\theta)\right)^{\prime} S_{r} \overline{\bar{J}} S_{r}^{\prime}\left(f\left(\theta_{0}\right)-f(\theta)\right)+o_{p}(1)+o_{p}^{*}(1) \text { in prob- } P,
\end{aligned}
$$

where $\overline{\bar{J}}$ is the bottom-right $q_{r} \times q_{r}$ submatrix of $\bar{J}$ and $o_{p}(1)$ and $o_{p}^{*}(1)$ are uniform in $\theta \in \Theta$. Let $A(\theta)$ and $B(\theta)$ denote the last two terms on the right-hand side of (45). Then

$$
\begin{aligned}
\sup _{\theta \in \Theta}|A(\theta)+B(\theta)| & \leq \sup _{\theta \in \Theta}|A(\theta)|+\sup _{\theta \in \Theta}|B(\theta)| \\
& =o_{p}(1)+o_{p}^{*}(1) \\
& =o_{p}^{*}(1) \text { in prob- } P
\end{aligned}
$$

where the last equality follows from Lemma B.1 of Dovonon and Gonçalves (2014) (see also Lemma 3 of Cheng and Huang, 2010). Therefore the consistency of $\widehat{\theta}_{o p t, T}$ follows from (45), (46), Assumptions (f) and (h)(1). This completes the proof of part (a) of Theorem 1.

To prove part (b), observe that the objective function for $\widehat{\theta}_{\text {diag, } T}$ is proportional to

$$
\left(\widehat{\gamma}_{T}-f(\theta)\right)^{\prime} W_{T}\left(\widehat{\gamma}_{T}-f(\theta)\right)=\left(f\left(\theta_{0}\right)-f(\theta)\right)^{\prime} W\left(f\left(\theta_{0}\right)-f(\theta)\right)+o_{p}^{*}(1) \text { in prob- } P,
$$

because of Assumptions (a), (b), (e) and (g). The consistency of $\widehat{\theta}_{\text {diag,T }}$ follows from (47) and Assumptions (f) and (g).

Proof of Theorem 2. It follows from Assumption (f), the first-order conditions and the 
mean value theorem that

$$
\begin{aligned}
\widehat{\theta}_{\text {opt }, T}-\theta_{0} & =\left(F\left(\widehat{\theta}_{\text {opt }, T}\right)^{\prime} \widehat{\Sigma}_{T}^{*-1} F\left(\widetilde{\theta}_{\text {opt }, T}\right)\right)^{-1} F\left(\widehat{\theta}_{\text {opt }, T}\right)^{\prime} \widehat{\Sigma}_{T}^{*-1}\left(\widehat{\gamma}_{T}-f\left(\theta_{0}\right)\right), \\
\widehat{\theta}_{\text {diag }, T}-\theta_{0} & =\left(F\left(\widehat{\theta}_{\text {diag }, T}\right)^{\prime} W_{T} F\left(\widetilde{\theta}_{\text {diag }, T}\right)\right)^{-1} F\left(\widehat{\theta}_{T}\right)^{\prime} W_{T}\left(\widehat{\gamma}_{T}-f\left(\theta_{0}\right)\right),
\end{aligned}
$$

where $\widetilde{\theta}_{o p t, T}$ and $\widetilde{\theta}_{\text {diag }, T}$ are points between $\widehat{\theta}_{\text {opt }, T}$ and $\theta_{0}$ and between $\widehat{\theta}_{\text {diag }, T}$ and $\theta_{0}$, respectively, implied by the mean value theorem.

It follows from (42) with $\theta=\theta_{0}$ and Assumptions (a)-(c) that

$$
\Upsilon_{T} S^{\prime}\left(\gamma\left(\bar{X}_{T}\right)-\gamma(\mu)\right) \stackrel{d}{\rightarrow}\left[\begin{array}{c}
S_{0}^{\prime} B_{0} Z \\
S_{1}^{\prime} B_{j_{1}}(Z \otimes \cdots \otimes Z) \\
\vdots \\
S_{r}^{\prime} B_{j_{r}}(Z \otimes \cdots \otimes Z)
\end{array}\right] \equiv \xi
$$

It follows from (44), Theorem 1(a), and Assumptions (c)-(e) that

$$
\begin{aligned}
& F\left(\widehat{\theta}_{o p t, T}\right)^{\prime} \widehat{\Sigma}_{T}^{*-1} F\left(\widetilde{\theta}_{o p t, T}\right) \\
= & F\left(\widehat{\theta}_{o p t, T}\right)^{\prime} S \Upsilon_{T}\left(\Upsilon_{T} S^{\prime} \widehat{\Sigma}_{T}^{*} S \Upsilon_{T}\right)^{-1} \Upsilon_{T} S^{\prime} F\left(\widetilde{\theta}_{o p t, T}\right) \\
= & {\left[T^{\frac{1}{2}} F_{0}^{\prime} S_{0} T^{\frac{j_{1}+1}{2}} F_{0}^{\prime} S_{1} \cdots T^{\frac{j_{r}+1}{2}} F_{0}^{\prime} S_{r}\right] J^{-1}\left[\begin{array}{c}
T^{\frac{1}{2}} S_{0}^{\prime} F_{0} \\
T^{\frac{j_{1}+1}{2}} S_{1}^{\prime} F_{0} \\
\vdots \\
T^{\frac{j_{r}+1}{2}} S_{r}^{\prime} F_{0}
\end{array}\right]+o_{p}^{*}\left(T^{j_{r}+1}\right) \text { in prob- } P } \\
= & T^{j_{r}+1} F_{0}^{\prime} S_{r} \overline{\bar{J}} S_{r}^{\prime} F_{0}+o_{p}^{*}\left(T^{j_{r}+1}\right) \text { in prob- } P
\end{aligned}
$$


and

$$
\begin{aligned}
& F\left(\widehat{\theta}_{o p t, T}\right)^{\prime} \widehat{\Sigma}_{T}^{*-1} S^{\prime-1} \Upsilon_{T}^{-1} \\
= & F\left(\widehat{\theta}_{o p t, T}\right)^{\prime} S \Upsilon_{T}\left(\Upsilon_{T} S^{\prime} \widehat{\Sigma}_{T}^{*} S \Upsilon_{T}\right)^{-1} \\
= & {\left[T^{\frac{1}{2}} F_{0}^{\prime} S_{0} T^{\frac{j_{1}+1}{2}} F_{0}^{\prime} S_{1} \cdots T^{\frac{j_{r}+1}{2}} F_{0}^{\prime} S_{r}\right] J^{-1}+o_{p}^{*}\left(T^{\frac{j_{r}+1}{2}}\right) \text { in prob- } P . }
\end{aligned}
$$

Combining (48), (50), (51) and (52), we obtain

$$
T^{\frac{j_{r}+1}{2}}\left(\widehat{\theta}_{o p t, T}-\theta_{0}\right) \stackrel{d^{*}}{\rightarrow}\left(F_{0}^{\prime} S_{r} \overline{\bar{J}} S_{r}^{\prime} F_{0}\right)^{-1} F_{0}^{\prime} S_{r} \bar{J} \xi \text { in prob- } P .
$$

Repeating similar arguments we obtain

$$
T^{\frac{j_{r}+1}{2}}\left(\widehat{\theta}_{o p t, T}^{*}-\widehat{\theta}_{o p t, T}\right) \stackrel{d^{* *}}{\rightarrow}\left(F_{0}^{\prime} S_{r} \overline{\bar{J}} S_{r}^{\prime} F_{0}\right)^{-1} F_{0}^{\prime} S_{r} \bar{J} \xi^{*} \text { in prob- } P^{*},
$$

which completes the proof of part (a).

Because

$$
T^{\frac{1}{2}}\left(\widehat{\gamma}_{T}-f\left(\theta_{0}\right)\right)=B_{0} Z_{T}+o_{p}(1),
$$

by Assumption (b), it follows from Assumption (a), (49) and (50) that

$$
\begin{aligned}
T^{\frac{1}{2}}\left(\widehat{\theta}_{\text {diag }, T}-\theta_{0}\right) & \stackrel{d^{*}}{\rightarrow}\left(F_{0}^{\prime} W F_{0}\right)^{-1} F_{0}^{\prime} W B_{0} Z \text { in prob- } P, \\
T^{\frac{1}{2}}\left(\widehat{\theta}_{\text {diag }, T}^{*}-\widehat{\theta}_{\text {diag }, T}\right) & \stackrel{d^{* *}}{\rightarrow}\left(F_{0}^{\prime} W F_{0}\right)^{-1} F_{0}^{\prime} W B_{0} Z^{*} \text { in prob- } P^{*},
\end{aligned}
$$


which completes the proof of part (b).

Proof of Theorem 3. It follows from the mean value theorem, (6), and Theorem 2(a) that

$$
\begin{aligned}
& \widehat{\Sigma}_{T}^{*-\frac{1}{2}}\left(\widehat{\gamma}_{T}-f\left(\widehat{\theta}_{o p t, T}\right)\right) \\
= & \left(\Upsilon_{T} S^{\prime} \widehat{\Sigma}_{T}^{*} S \Upsilon_{T}\right)^{-\frac{1}{2}} \Upsilon_{T} S^{\prime}\left(\widehat{\gamma}_{T}-f\left(\widehat{\theta}_{o p t, T}\right)\right) \\
= & \left(\Upsilon_{T} S^{\prime} \widehat{\Sigma}_{T}^{*} S \Upsilon_{T}\right)^{-\frac{1}{2}} \Upsilon_{T} S^{\prime}\left(\widehat{\gamma}_{T}-f\left(\theta_{0}\right)\right)-\left(\Upsilon_{T} S^{\prime} \widehat{\Sigma}_{T}^{*} S \Upsilon_{T}\right)^{-\frac{1}{2}} \Upsilon_{T} S^{\prime} F\left(\widetilde{\theta}_{o p t, T}\right)\left(\widehat{\theta}_{o p t, T}-\theta_{0}\right) \\
= & J^{-\frac{1}{2}} \xi-J^{-\frac{1}{2}} S_{r}^{\prime} F_{0}\left(F_{0}^{\prime} S_{r} \overline{\bar{J}} S_{r}^{\prime} F_{0}\right)^{-1} F_{0}^{\prime} S_{r} \bar{J} \xi+o_{p}^{*}(1) \text { in prob-P,}
\end{aligned}
$$

where $\tilde{\theta}_{o p t, T}$ is a point between $\widehat{\theta}_{\text {opt }, T}$ and $\theta_{0}$ and $A^{\frac{1}{2}}$ is the matrix such that $A^{\frac{1}{2}} A^{\frac{1}{2}}=A$. Thus it follows from (56) that

$$
J_{T} \stackrel{d^{*}}{\rightarrow} \eta^{\prime} \eta \text { in prob- } P \text {. }
$$

The bootstrap version of this result can be derived in a similar way.

Proof of Theorem 4. We follow the steps in the proof of Theorem 1 in Chernozhukov and Hong (2003) by showing that

$$
\int_{H_{T}}\|h\|^{\alpha}\left|p_{T}^{*}(h)-p_{\infty}^{*}(h)\right| d h=o_{p}^{*}(1) \text { in prob- } P
$$

for every $\alpha \geq 0$, from which Theorem 4 immediately follows. It is convenient to write the localized quasi-posterior as

$$
\begin{aligned}
p_{T}^{*}(h) & =p_{T}\left(\theta_{0}+\frac{h}{T^{\frac{j r+1}{2}}}-\left(\nabla^{2} q_{T}\left(\theta_{0}\right)\right)^{-1} \nabla q_{T}\left(\theta_{0}\right)\right) \\
& =\frac{\pi\left(\theta_{0}+\frac{h}{T^{\frac{j r+1}{2}}}-\left(\nabla^{2} q_{T}\left(\theta_{0}\right)\right)^{-1} \nabla q_{T}\left(\theta_{0}\right)\right) \exp \left(-q_{T}\left(\theta_{0}+\frac{h}{T^{\frac{j r+1}{2}}}-\left(\nabla^{2} q_{T}\left(\theta_{0}\right)\right)^{-1} \nabla q_{T}\left(\theta_{0}\right)\right)\right)}{\int_{H_{T}} \pi\left(\theta_{0}+\frac{h}{T^{\frac{j r+1}{2}}}-\left(\nabla^{2} q_{T}\left(\theta_{0}\right)\right)^{-1} \nabla q_{T}\left(\theta_{0}\right)\right) \exp \left(-q_{T}\left(\theta_{0}+\frac{h}{T^{\frac{j r+1}{2}}}-\left(\nabla^{2} q_{T}\left(\theta_{0}\right)\right)^{-1} \nabla q_{T}\left(\theta_{0}\right)\right)\right) d h} \\
& =\frac{\pi\left(\theta_{0}+\frac{h}{T^{\frac{j r+1}{2}}}-\left(\nabla^{2} q_{T}\left(\theta_{0}\right)\right)^{-1} \nabla q_{T}\left(\theta_{0}\right)\right) \exp (\omega(h))}{\int_{H_{T}} \pi\left(\theta_{0}+\frac{h}{T^{\frac{j r+1}{2}}}-\left(\nabla^{2} q_{T}\left(\theta_{0}\right)\right)^{-1} \nabla q_{T}\left(\theta_{0}\right)\right) \exp (\omega(h)) d h}
\end{aligned}
$$


where

$\omega(h)=-q_{T}\left(\theta_{0}+\frac{h}{T^{\frac{j_{r}+1}{2}}}-\left(\nabla^{2} q_{T}\left(\theta_{0}\right)\right)^{-1} \nabla q_{T}\left(\theta_{0}\right)\right)+q_{T}\left(\theta_{0}\right)+\frac{1}{2} \nabla q_{T}\left(\theta_{0}\right)^{\prime}\left(\nabla^{2} q_{T}\left(\theta_{0}\right)\right)^{-1} \nabla q_{T}\left(\theta_{0}\right)$.

To prove (58), we first show that

$$
\begin{aligned}
\int_{H_{T}}\|h\|^{\alpha} \mid \pi\left(\theta_{0}+\frac{h}{T^{\frac{j r}{2}+1}}\right. & \left.-\left(\nabla^{2} q_{T}\left(\theta_{0}\right)\right)^{-1} \nabla q_{T}\left(\theta_{0}\right)\right) \exp (\omega(h)) \\
& -\pi\left(\theta_{0}\right) \exp \left(-\frac{1}{2} h^{\prime} F_{0}^{\prime} S_{r} \overline{\bar{J}} S_{r}^{\prime} F_{0} h\right) \mid d h=o_{p}^{*}(1) \text { in prob- } P(60)
\end{aligned}
$$

Using the second-order Taylor series approximation, $\omega(h)$ can be written as

$$
\begin{aligned}
\omega(h)= & -\nabla q_{T}\left(\theta_{0}\right)^{\prime}\left(\frac{h}{T^{\frac{j_{r}+1}{2}}}-\left(\nabla^{2} q_{T}\left(\theta_{0}\right)\right)^{-1} \nabla q_{T}\left(\theta_{0}\right)\right) \\
& -\frac{1}{2}\left(\frac{h}{T^{\frac{j_{r}+1}{2}}}-\left(\nabla^{2} q_{T}\left(\theta_{0}\right)\right)^{-1} \nabla q_{T}\left(\theta_{0}\right)\right)^{\prime} \nabla^{2} q_{T}\left(\theta_{0}\right)\left(\frac{h}{T^{\frac{j_{r}+1}{2}}}-\left(\nabla^{2} q_{T}\left(\theta_{0}\right)\right)^{-1} \nabla q_{T}\left(\theta_{0}\right)\right) \\
& \left.-\frac{1}{2} \nabla q_{T}\left(\theta_{0}\right)^{\prime} \nabla^{2} q_{T}\left(\theta_{0}\right)\right]^{-1} \nabla q_{T}\left(\theta_{0}\right)+R_{T}\left(\frac{h}{T^{\frac{j_{r}+1}{2}}}-\left(\nabla^{2} q_{T}\left(\theta_{0}\right)\right)^{-1} \nabla q_{T}\left(\theta_{0}\right)\right) \\
= & -\frac{1}{2 T^{j_{r}+1}} h^{\prime} \nabla^{2} q_{T}\left(\theta_{0}\right) h+R_{T}\left(\frac{h}{T^{\frac{j_{r}+1}{2}}}-\left(\nabla^{2} q_{T}\left(\theta_{0}\right)\right)^{-1} \nabla q_{T}\left(\theta_{0}\right)\right)
\end{aligned}
$$

where $R_{T}(\cdot)$ is the remainder term. Note that the integral in (60) can be written as the sum of three integrals over (i) $\left\{h \in H_{T}:\|h\| \leq M\right\}$, (ii) $\left\{h \in H_{T}: M \leq\|h\| \leq \delta T^{j_{r}+1}\right\}$ and (iii) $\left\{h \in H_{T}:\|h\| \geq \delta T^{j_{r}+1}\right\}$. We evaluate each of the three integrals in turn.

Start with the integral over set (i). It follows from the continuity of $\pi(\cdot)$, the smoothness of $f(\cdot),(51)$ and $(52)$ that

$$
\begin{array}{r}
\sup _{\|h\| \leq M}\left|\pi\left(\theta_{0}+\frac{h}{T^{\frac{j_{r}+1}{2}}}-\left(\nabla^{2} q_{T}\left(\theta_{0}\right)\right)^{-1} \nabla q_{T}\left(\theta_{0}\right)\right)-\pi\left(\theta_{0}\right)\right| \stackrel{p}{\rightarrow} 0, \\
\sup _{\|h\| \leq M}\left|R_{T}\left(\frac{h}{T^{\frac{j_{r}+1}{2}}}-\left(\nabla^{2} q_{T}\left(\theta_{0}\right)\right)^{-1} \nabla q_{T}\left(\theta_{0}\right)\right)\right| \stackrel{p}{\rightarrow} 0,
\end{array}
$$


from which it follows that

$$
\begin{aligned}
& \sup _{\|h\| \leq M}\|h\|^{\alpha}\left|\pi\left(\theta_{0}+\frac{h}{T^{\frac{j_{r}+1}{2}}}-\left(\nabla^{2} q_{T}\left(\theta_{0}\right)\right)^{-1} \nabla q_{T}\left(\theta_{0}\right)\right) \exp (\omega(h))-\pi\left(\theta_{0}\right) \exp \left(-\frac{1}{2} h^{\prime} \nabla^{2} q_{T}\left(\theta_{0}\right) h\right)\right| \\
= & o_{p}^{*}(1) \text { in prob- } P .
\end{aligned}
$$

Hence, we have that for every $0<M<\infty$ and every $\varepsilon>0$,

$$
\begin{aligned}
& \quad \liminf _{T} P_{*}\left(\int_{h \in H_{T}:\|h\| \leq M}\|h\|^{\alpha} \mid \pi\left(\theta_{0}+\frac{h}{T^{\frac{j_{r}+1}{2}}}-\left(\nabla^{2} q_{T}\left(\theta_{0}\right)\right)^{-1} \nabla q_{T}\left(\theta_{0}\right)\right) \exp (\omega(h))\right. \\
& \left.\quad-\pi\left(\theta_{0}\right) \exp \left(-\frac{1}{2} h^{\prime} F_{0}^{\prime} S_{r} \overline{\bar{J}} S_{r}^{\prime} F_{0} h\right) \mid\right) \\
& \geq 1-\varepsilon .
\end{aligned}
$$

Thus the integral over set (i) is $o_{p}(1)$.

Next consider the integral over set (ii). Note that

$$
\left.\left.\int_{M<\|h\|<\delta T^{\frac{j_{r}+1}{2}}} \mid \pi\left(\theta_{0}\right) \exp \left(-\frac{1}{2} h^{\prime} F_{0}^{\prime} S_{r} \overline{\bar{J}} S_{r}^{\prime} F_{0} h\right)\right)\right) \mid d h
$$

can be made arbitrarily small by choosing sufficiently large $M$. Using the quadratic approximation of $\omega(h),(61)$, we can write

$$
\exp (\omega(h)) \leq \exp \left(-\frac{1}{2} h^{\prime} \nabla^{2} q_{T}\left(\theta_{0}\right) h+\left|R_{T}\left(\frac{h}{T^{\frac{j_{r}+1}{2}}}-\left(\nabla^{2} q_{T}\left(\theta_{0}\right)\right)^{-1} \nabla q_{T}\left(\theta_{0}\right)\right)\right|\right)
$$

Because $f$ is twice continuously differentiable, Assumption 4(iv)(a) of Chernozhukov and Hong (2003) is satisfied. Thus, for every $\varepsilon>0$, there are some $\delta>0$ and $M>0$ such that $\liminf P_{*}\left(\sup _{M \leq\|h\| \leq \delta T^{\frac{j_{r}+1}{2}}} \frac{\left|R_{T}\left(\frac{h}{T^{\frac{j_{r}+1}{2}}}-\left(\nabla^{2} q_{T}\left(\theta_{0}\right)\right)^{-1} \nabla q_{T}\left(\theta_{0}\right)\right)\right|}{\left\|h-T^{\frac{j_{r}+1}{2}}\left(\nabla^{2} q_{T}\left(\theta_{0}\right)\right)^{-1} \nabla q_{T}\left(\theta_{0}\right)\right\|^{2}} \leq \frac{1}{4} \operatorname{maxeig}\left(\nabla^{2} q_{T}\left(\theta_{0}\right)\right)\right) \geq 1-\varepsilon$

Because $\left(\nabla^{2} q_{T}\left(\theta_{0}\right)\right)^{-1} \nabla q_{T}\left(\theta_{0}\right)=O_{p}(1)$, it follows from (67) and (68) that there is $C$ such 
that

$$
\liminf _{T} P_{*}\left(\exp (\omega(h)) \leq C \exp \left(-\frac{1}{4} h^{\prime} \nabla^{2} q_{T}\left(\theta_{0}\right) h\right)\right) \geq 1-\varepsilon
$$

Combining these results, it follows that

$\liminf _{T} P_{*}\left(\int_{h \in H_{T}: M<\|h\|<\delta T^{\frac{j r+1}{2}}}\|h\|^{\alpha}\left|\pi\left(\theta_{0}+\frac{h}{T^{\frac{j_{r}+1}{2}}}-\left(\nabla^{2} q_{T}\left(\theta_{0}\right)\right)^{-1} \nabla q_{T}\left(\theta_{0}\right)\right) \exp (\omega(h)) d h<\varepsilon\right|\right) \geq 1-\varepsilon$

from which we obtain

$$
\begin{aligned}
& \quad \liminf _{T} P_{*}\left(\int_{h \in H_{T}: M<\|h\|<\delta T^{\frac{j_{r}+1}{2}}}\|h\|^{\alpha} \mid \pi\left(\theta_{0}+\frac{h}{T^{\frac{j_{r}+1}{2}}}-\left(\nabla^{2} q_{T}\left(\theta_{0}\right)\right)^{-1} \nabla q_{T}\left(\theta_{0}\right)\right) \exp (\omega(h))\right. \\
& \left.\quad-\pi\left(\theta_{0}\right) \exp \left(-\frac{1}{2} h^{\prime} \nabla^{2} q_{T}\left(\theta_{0}\right) h\right) \mid\right) \\
& \geq 1-\varepsilon .
\end{aligned}
$$

Thus, the integral over set (ii) is also asymptotically negligible.

Third, consider the integral over set (iii). As in the second integral,

$$
\left.\left.\int_{\|h\| \geq \delta T^{\frac{j_{r}+1}{2}}} \mid \pi\left(\theta_{0}\right) \exp \left(-\frac{1}{2} h^{\prime} F_{0}^{\prime} S_{r} \overline{\bar{J}} S_{r}^{\prime} F_{0} h\right)\right)\right) \mid d h
$$

goes to zero on set (iii). Note that

$$
\int_{h \in H_{T}:\|h\| \geq \delta T^{\frac{j_{r}+1}{2}}}\|h\|^{\alpha} \exp (\omega(h)) \pi\left(\theta_{0}+\frac{h}{T^{\frac{j_{r}+1}{2}}}-\left(\nabla^{2} q_{T}\left(\theta_{0}\right)\right)^{-1} \nabla q_{T}\left(\theta_{0}\right)\right)=o_{p}^{*}(1) \text { in prob- } P
$$

is bounded by

$$
\begin{aligned}
& T^{\frac{\left(j_{r}+1\right) \alpha}{2}+1} \int_{\left\|\theta-\theta_{0}+\left(\nabla^{2} q_{T}\left(\theta_{0}\right)\right)^{-1} \nabla q_{T}\left(\theta_{0}\right)\right\| \geq \delta}\left\|\theta-\theta_{0}+\left(\nabla^{2} q_{T}\left(\theta_{0}\right)\right)^{-1} \nabla q_{T}\left(\theta_{0}\right)\right\|^{\alpha} \pi(\theta) \\
& \times \exp \left(q_{T}\left(\theta_{0}\right)-q_{T}(\theta)-\frac{1}{2} \nabla q_{T}\left(\theta_{0}\right)^{\prime}\left(\nabla^{2} q_{T}\left(\theta_{0}\right)\right)^{-1} \nabla q_{T}\left(\theta_{0}\right)\right) d \theta .
\end{aligned}
$$


Since

$$
\left(\nabla^{2} q_{T}\left(\theta_{0}\right)\right)^{-1} \nabla q_{T}\left(\theta_{0}\right)=o_{p}^{*}(1) \text { in prob- } P
$$

(74) is in turn bounded by

$C \exp \left(-\frac{1}{2} \nabla q_{T}\left(\theta_{0}\right)^{\prime}\left(\nabla^{2} q_{T}\left(\theta_{0}\right)\right)^{-1} \nabla q_{T}\left(\theta_{0}\right)\right) T^{1+\frac{\alpha\left(j_{r}+1\right)}{2}} \int_{\left\|\theta-\theta_{0}\right\| \geq \delta}\left(1+\|\theta\|^{\alpha}\right) \pi(\theta) \exp \left(q_{T}\left(\theta_{0}\right)-q_{T}(\theta)\right) d \theta$

for some $C>0$. It follows from equation (45) that there is $\varepsilon>0$ such that

$$
\liminf _{T \rightarrow \infty} P_{*}\left(\sup _{\left\|\theta-\theta_{0}\right\| \geq \delta / 2} \exp \left(q_{T}\left(\theta_{0}\right)-q_{T}(\theta)\right) \leq \exp (-T \epsilon)\right)=1 .
$$

Hence with probability approaching one (76) is bounded by

$$
\begin{aligned}
& C \exp \left(-\frac{1}{2} \nabla q_{T}\left(\theta_{0}\right)^{\prime}\left(\nabla^{2} q_{T}\left(\theta_{0}\right)\right)^{-1} \nabla q_{T}\left(\theta_{0}\right)\right) T^{1+\frac{\alpha\left(j_{r}+1\right)}{2}} \exp (-T \varepsilon) \int_{\Theta}\|\theta\|^{\alpha} \pi(\theta) d \theta \\
= & o_{p}^{*}(1) \text { in prob- } P .
\end{aligned}
$$

Thus we have

$$
\begin{aligned}
& \liminf _{T} P_{*}\left\{\int_{h \in H_{T}:\|h\| \geq \delta T}\|h\|^{\alpha} \exp (\omega(h)) \pi\left(\theta_{0}+\frac{h}{T^{\frac{j_{r}+1}{2}}}-\left(\nabla^{2} q_{T}\left(\theta_{0}\right)\right)^{-1} \nabla q_{T}\left(\theta_{0}\right)\right)\right. \\
& \left.\left.-\exp \left(-\frac{1}{2} h^{\prime} F_{0}^{\prime} S_{r} \overline{\bar{J}} S_{r}^{\prime} F_{0} h\right)\right) \pi\left(\theta_{0}\right) \mid d h<\varepsilon\right) \geq 1-\varepsilon
\end{aligned}
$$


In other words, the integral over set (iii) also converges in probability to zero.

Equation (60) follows from (65), (71) and (79). It follows from (60) with $\alpha=0$ that

$$
\begin{aligned}
\int_{H_{T}} D_{T} d h & \equiv \pi\left(\theta_{0}+\frac{h}{T^{\frac{j_{r}+1}{2}}}-\left(\nabla^{2} q_{T}\left(\theta_{0}\right)\right)^{-1} \nabla q_{T}\left(\theta_{0}\right)\right) \exp (\omega(h)) d h \\
& =\int_{H_{T}} \pi\left(\theta_{0}\right) \exp \left(-\frac{1}{2 T^{2}} h^{\prime} \nabla^{2} q_{T}\left(\theta_{0}\right) h\right) d h+o_{p}^{*}(1) \text { in prob- } P \\
& =\int_{H_{T}} \pi\left(\theta_{0}\right) \exp \left(-\frac{1}{2} h^{\prime} F_{0}^{\prime} S_{r} \overline{\bar{J}} S_{r}^{\prime} F_{0} h\right) d h+o_{p}^{*}(1) \text { in prob- } P \\
& =\int_{\Re^{p}} \pi\left(\theta_{0}\right) \exp \left(-\frac{1}{2} h^{\prime} F_{0}^{\prime} S_{r} \overline{\bar{J}} S_{r}^{\prime} F_{0} h\right) d h+o_{p}^{*}(1) \text { in prob- } P \\
& =\pi\left(\theta_{0}\right)(2 \pi)^{\frac{l}{2}}\left|F_{0}^{\prime} S_{r} \overline{\bar{J}} S_{r}^{\prime} F_{0}\right|^{-\frac{1}{2}}+o_{p}^{*}(1) \text { in prob- } P .
\end{aligned}
$$

Thus it follows from (60) and (80) that

$$
\begin{aligned}
& \int_{H_{T}}\|h\|^{\alpha}\left|p_{T}^{*}(h)-p_{\infty}^{*}(h)\right| d h \\
= & \frac{1}{D_{T}} \int_{H_{T}}\|h\|^{\alpha} \mid \pi\left(\theta_{0}+\frac{h}{T^{\frac{j r+1}{2}}}-\left(\nabla^{2} q_{T}\left(\theta_{0}\right)\right)^{-1} \nabla q_{T}\left(\theta_{0}\right)\right) \exp (\omega(h)) \\
& -D_{T}\left(\frac{\left|F_{0}^{\prime} S_{r} \overline{\bar{J}} S_{r}^{\prime} F_{0}\right|}{(2 \pi)^{l}}\right)^{\frac{1}{2}} \exp \left(-\frac{1}{2} h^{\prime} F_{0}^{\prime} S_{r} \overline{\bar{J}} S_{r}^{\prime} F_{0} h\right) \mid d h \\
= & o_{p}^{*}(1) \text { in prob- } P
\end{aligned}
$$

from which (58) follows. This completes the proof of Theorem 4.

Proof of the Proposition. The proof follows from results in Inoue and Kilian (2016), but is included here for completeness. Recall the definition of $S_{j}$ and $S$. It follows from (50) that the limiting covariance matrix of $\Upsilon_{T} S^{\prime}\left(\gamma\left(\bar{X}_{T}\right)-\gamma(\mu)\right)$ is given by $J$ in Assumption (c).

Similarly, the limiting covariance matrix of $\Upsilon_{T} S^{\prime}\left(\gamma\left(\bar{X}_{T}^{*}\right)-\gamma\left(\bar{X}_{T}\right)\right)$ is also $J$. The first part of the Proposition follows from Theorem 1, (43), and (50). The proof of the second part is analogous. 


\section{Appendix B: Illustrative examples}

Verifying the high-level Assumption (c) may require tedious algebra and the use of numerical derivatives. This appendix contains two stylized examples for which the nonsingularity of $J$ may be verified analytically.

Example 1:

First, consider the $\mathrm{AR}(1)$ process

$$
y_{t}=\rho y_{t-1}+u_{t}
$$

where $|\rho|<1$ and $u_{t} \stackrel{\text { iid }}{\sim}\left(0, \sigma^{2}\right)$ with $E\left(u_{t}^{3}\right)=0$ and finite fourth moments. Consider testing the joint null hypothesis that $\rho=\rho_{0}$ and $\rho^{2}=\rho_{0}^{2}$, where $\rho$ and $\rho^{2}$ correspond to the responses of $y_{t+h}$ to a unit shock in $u_{t}$ for $h=1,2$. Suppose that

$$
\left[\begin{array}{c}
T^{-\frac{1}{2}} \sum_{t=2}^{T} y_{t-1} u_{t} \\
T^{-\frac{1}{2}} \sum_{t=2}^{T}\left(y_{t-1}^{2}-E\left(y_{t-1}^{2}\right)\right)
\end{array}\right] \stackrel{d}{\rightarrow} N\left(\left[\begin{array}{l}
0 \\
0
\end{array}\right],\left[\begin{array}{cc}
\omega_{11} & \omega_{12} \\
\omega_{21} & \omega_{22}
\end{array}\right]\right)
$$

Let $Z_{T}=\left[Z_{1 T} Z_{2 T}\right]^{\prime}$,

$$
\begin{aligned}
& Z_{1 T}=\frac{1}{\sqrt{\omega_{11} T}} \sum_{t=2}^{T} y_{t-1} u_{t} \\
& Z_{2 T}=\frac{1}{\kappa \sqrt{T}}\left[\sum_{t=2}^{T}\left(y_{t-1}^{2}-E\left(y_{t-1}^{2}\right)\right)-\frac{\omega_{12}}{\omega_{11}} \sum_{t=2}^{T} y_{t-1} u_{t}\right],
\end{aligned}
$$

where $\kappa=\left(\omega_{22}-\omega_{12}^{2} / \omega_{22}\right)^{1 / 2}$. Note that $Z_{T} \stackrel{d}{\rightarrow} N\left(0_{2 \times 1}, I_{2}\right)$ and

Given the second-order Taylor series expansion

$$
\begin{aligned}
\widehat{\rho}_{T}-\rho & =\frac{\sum_{t=2}^{T} y_{t-1} u_{t}}{\sum_{t=2}^{T} y_{t-1}^{2}} \\
& =\frac{1}{E\left(y_{t-1}^{2}\right)} \frac{1}{T} \sum_{t=2}^{T} y_{t-1} u_{t}-\frac{1}{\left[E\left(y_{t-1}^{2}\right)\right]^{2}} \frac{1}{T} \sum_{t=2}^{T} y_{t-1} u_{t} \frac{1}{T} \sum_{s=2}^{T}\left(y_{s-1}^{2}-E\left(y_{s-1}^{2}\right)\right)+o_{p}\left(\frac{1}{T}\right)
\end{aligned}
$$




$$
\begin{aligned}
& =\frac{1-\rho^{2}}{\sigma^{2}} \frac{1}{T} \sum_{t=2}^{T} y_{t-1} u_{t}-\frac{\left(1-\rho^{2}\right)^{2}}{\sigma^{4}} \frac{1}{T} \sum_{t=2}^{T} y_{t-1} u_{t} \frac{1}{T} \sum_{s=2}^{T}\left(y_{s-1}^{2}-E\left(y_{s-1}^{2}\right)\right)+o_{p}\left(\frac{1}{T}\right) \\
& =\frac{1}{\sqrt{T}}\left(1-\rho^{2}\right)^{\frac{1}{2}} Z_{1 T}+\frac{1}{T}\left(\frac{\left(1-\rho^{2}\right)^{\frac{3}{2}} \omega_{12}}{\sigma^{2} \omega_{11}} Z_{1 T}^{2}+\frac{\left(1-\rho^{2}\right)^{\frac{3}{2}} \kappa}{\sigma^{2}} Z_{1 T} Z_{2 T}\right)+o_{p}\left(\frac{1}{T}\right)
\end{aligned}
$$

and

$$
\widehat{\rho}_{T}^{2}-\rho^{2}=2 \rho\left(\widehat{\rho}_{T}-\rho\right)+\left(\widehat{\rho}_{T}-\rho\right)^{2},
$$

we can write

$$
\begin{aligned}
& {\left[\begin{array}{c}
\widehat{\rho}_{T}-\rho \\
\widehat{\rho}_{T}^{2}-\rho^{2}
\end{array}\right]} \\
& =T^{-\frac{1}{2}}\left[\begin{array}{cc}
\left(1-\rho^{2}\right)^{\frac{1}{2}} & 0 \\
2 \rho\left(1-\rho^{2}\right)^{\frac{1}{2}} & 0
\end{array}\right] Z_{T} \\
& +T^{-1}\left[\begin{array}{cccc}
\frac{\left(1-\rho^{2}\right)^{\frac{3}{2}} \omega_{12}}{\sigma^{2} \omega_{11}} & \frac{\left(1-\rho^{2}\right)^{\frac{3}{2}} \kappa}{2 \sigma^{2}} & \frac{\left(1-\rho^{2}\right)^{\frac{3}{2}} \kappa}{2 \sigma^{2}} & 0 \\
1-\rho^{2}+\frac{2 \rho\left(1-\rho^{2}\right)^{\frac{3}{2}} \omega_{12}}{\sigma^{2} \omega_{11}} & \frac{\rho\left(1-\rho^{2}\right)^{\frac{3}{2}} \kappa}{\sigma^{2}} & \frac{\rho\left(1-\rho^{2}\right)^{\frac{3}{2}} \kappa}{\sigma^{2}} & 0
\end{array}\right]\left(Z_{T} \otimes Z_{T}\right) \\
& +\left[\begin{array}{c}
o_{p}\left(T^{-1}\right) \\
o_{p}\left(T^{-1}\right)
\end{array}\right] \text {. }
\end{aligned}
$$

By the Schur decomposition theorem, we have

$$
S^{\prime} B_{0} B_{0}^{\prime} S=\Lambda,
$$

where

$S=\frac{1}{\left(4 \rho^{2}+1\right)^{\frac{1}{2}}}\left[\begin{array}{cc}1 & 2 \rho \\ 2 \rho & -1\end{array}\right], \quad B_{0}=\left[\begin{array}{cc}\left(1-\rho^{2}\right)^{\frac{1}{2}} & 0 \\ 2 \rho\left(1-\rho^{2}\right)^{\frac{1}{2}} & 0\end{array}\right], \quad \Lambda=\left[\begin{array}{cc}\left(1-\rho^{2}\right)\left(4 \rho^{2}+1\right) & 0 \\ 0 & 0\end{array}\right]$

Thus it can be shown that 


$$
\begin{aligned}
& \Upsilon_{T} S^{\prime}\left[\begin{array}{c}
\widehat{\rho}_{T}-\rho \\
\widehat{\rho}_{T}^{2}-\rho^{2}
\end{array}\right] \\
& =\left[\begin{array}{cc}
{\left[\left(1-\rho^{2}\right)\left(4 \rho^{2}+1\right)\right]^{\frac{1}{2}}} & 0 \\
0 & 0
\end{array}\right] Z_{T} \\
& +\left[\begin{array}{cccc}
\frac{1}{\sqrt{T}} \frac{2 \rho\left(1-\rho^{2}\right)}{\left(4 \rho^{2}+1\right)^{\frac{1}{2}}}+\frac{1}{\sqrt{T}} \frac{\left(4 \rho^{2}+1\right)^{\frac{1}{2}}\left(1-\rho^{2}\right)^{\frac{3}{2}} \omega_{12}}{\sigma^{2} \omega_{11}} & \frac{\left(4 \rho^{2}+1\right)^{\frac{1}{2}}\left(1-\rho^{2}\right)^{\frac{3}{2}} \kappa}{2 \sigma^{2} T^{\frac{1}{2}}} & \frac{\left(4 \rho^{2}+1\right)^{\frac{1}{2}}\left(1-\rho^{2}\right)^{\frac{3}{2}} \kappa}{2 \sigma^{2} T^{\frac{1}{2}}} & 0 \\
-\frac{1-\rho^{2}}{\left(4 \rho^{2}+1\right)^{\frac{1}{2}}} & 0 & 0 & 0
\end{array}\right]\left(Z_{T} \otimes Z_{T}\right) \\
& +o_{p}(1) \\
& =\left[\left(1-\rho^{2}\right)\left(4 \rho^{2}+1\right)\right]^{\frac{1}{2}} Z_{1 T}-\frac{1-\rho^{2}}{\left(4 \rho^{2}+1\right)^{\frac{1}{2}}} Z_{1 T}^{2}+o_{p}(1),
\end{aligned}
$$

where

$$
\Upsilon_{T}=\left[\begin{array}{cc}
T^{\frac{1}{2}} & 0 \\
0 & T
\end{array}\right]
$$

Note that the first element of (92) converges in distribution to a normal random variable, whereas the second element converges in distribution to a chi-square random variable up to scale.

$$
J=\left[\begin{array}{cc}
\left(1-\rho^{2}\right)\left(4 \rho^{2}+1\right) & 0 \\
0 & \frac{3\left(1-\rho^{2}\right)^{2}}{4 \rho^{2}+1}
\end{array}\right],
$$

is nonsingular as required by Assumption (c).

\section{Example 2:}

Next, consider the stationary bivariate VAR(1) process

$$
y_{t}=\Phi y_{t-1}+u_{t}
$$


where

$$
\Phi=\left[\begin{array}{cc}
\phi_{1} & 0 \\
0 & \phi_{2}
\end{array}\right], \quad u_{t} \stackrel{i i d}{\sim}\left(0_{2 \times 1}, \Sigma\right), \quad \Sigma=\left[\begin{array}{cc}
\sigma_{1}^{2} & 0 \\
0 & \sigma_{2}^{2}
\end{array}\right] .
$$

Suppose $\sigma_{1}^{2} /\left(1-\phi_{1}^{2}\right)=\sigma_{2} /\left(1-\phi_{2}\right)=1$ so that

$$
E\left(y_{t} y_{t}^{\prime}\right)=I_{2}
$$

Let $\widehat{\Phi}=\left(\sum_{t=2}^{T} y_{t-1} y_{t-1}^{-1} \sum_{t=2}^{T} y_{t-1} y_{t}\right.$ and consider the joint distribution of

$$
\left[\operatorname{vec}(\widehat{\Phi}-\Phi)^{\prime} \operatorname{vec}\left(\widehat{\Phi}^{2}-\Phi^{2}\right)^{\prime}\right]^{\prime}
$$

It follows from equation (96) that

$$
\begin{aligned}
\widehat{\Phi}-\Phi= & \left(\frac{1}{T} \sum_{t=2}^{T} y_{t-1} y_{t-1}^{\prime}\right)^{-1} \frac{1}{T} \sum_{t=2}^{T} y_{t-1} u_{t} \\
= & \left(E \left(y_{t-1} y_{t-1}^{\prime-1} \frac{1}{\sqrt{T}} \sum_{t=2}^{T} y_{t-1} u_{t}\right.\right. \\
& +\left(\frac{1}{T} \sum_{t=2}^{T}\left(y_{t-1} y_{t-1}^{\prime}-E\left(y_{t-1} y_{t-1}^{\prime}\right)\right)\right)^{-1} \frac{1}{T} \sum_{t=2}^{T} y_{t-1} u_{t}+o_{p}\left(T^{-1}\right) \\
= & \frac{1}{T} \sum_{t=2}^{T} y_{t-1} u_{t}+\left(\frac{1}{T} \sum_{t=2}^{T}\left(y_{t-1} y_{t-1}^{\prime}-E\left(y_{t-1} y_{t-1}^{\prime}\right)\right)\right)^{-1} \frac{1}{T} \sum_{t=2}^{T} y_{t-1} u_{t}+o_{p}\left(T^{-1}\right) .
\end{aligned}
$$

Similarly,

$$
\begin{aligned}
\widehat{\Phi}^{2}-\Phi^{2}= & \Phi \frac{1}{T} \sum_{t=2}^{T} y_{t-1} u_{t}^{\prime}+\frac{1}{T} \sum_{t=2}^{T} y_{t-1} u_{t}^{\prime} \Phi+\left(\frac{1}{T} \sum_{t=2}^{T} y_{t-1} u_{t}^{\prime}\right)^{2} \\
& -\Phi\left(\frac{1}{T} \sum_{t=2}^{T}\left(y_{t-1} y_{t-1}^{\prime}-E\left(y_{t-1} y_{t-1}^{\prime}\right)\right)\right)^{-1} \frac{1}{T} \sum_{t=2}^{T} y_{t-1} u_{t} \\
& -\left(\frac{1}{T} \sum_{t=2}^{T}\left(y_{t-1} y_{t-1}^{\prime}-E\left(y_{t-1} y_{t-1}^{\prime}\right)\right)\right)^{-1} \frac{1}{T} \sum_{t=2}^{T} y_{t-1} u_{t} \Phi
\end{aligned}
$$


After some tedius matrix algebra, it follows from (97) and (98) that

$$
\left[\begin{array}{c}
\operatorname{vec}(\widehat{\Phi}-\Phi) \\
\operatorname{vec}\left(\widehat{\Phi}^{2}-\Phi^{2}\right)
\end{array}\right]=J_{1} \Omega^{\frac{1}{2}} Z_{T}+\frac{1}{2} J_{2}\left(\Omega^{\frac{1}{2}} \otimes \Omega^{\frac{1}{2}}\right)\left(Z_{T} \otimes Z_{T}\right)+o_{p}\left(T^{-1}\right)
$$

where

$$
J_{1}=\left[\begin{array}{cc}
I_{4} & 0_{4 \times 3} \\
I_{2} \otimes \Phi+\Phi^{\prime} \otimes I_{2} & 0_{4 \times 4}
\end{array}\right]
$$

and $\Omega$ is the asymptotic covariance matrix of $\left[\operatorname{vec}\left(T^{-1 / 2} \sum_{t=2}^{T} y_{t-1} u_{t}^{\prime}\right)^{\prime} \operatorname{vech}\left(T^{-1 / 2} \sum_{t=2}^{T} y_{t-1} y_{t-1}^{\prime}\right)^{\prime}\right]^{\prime}$. 
$J_{2}$ is given by the following $8 \times 49$ matrix

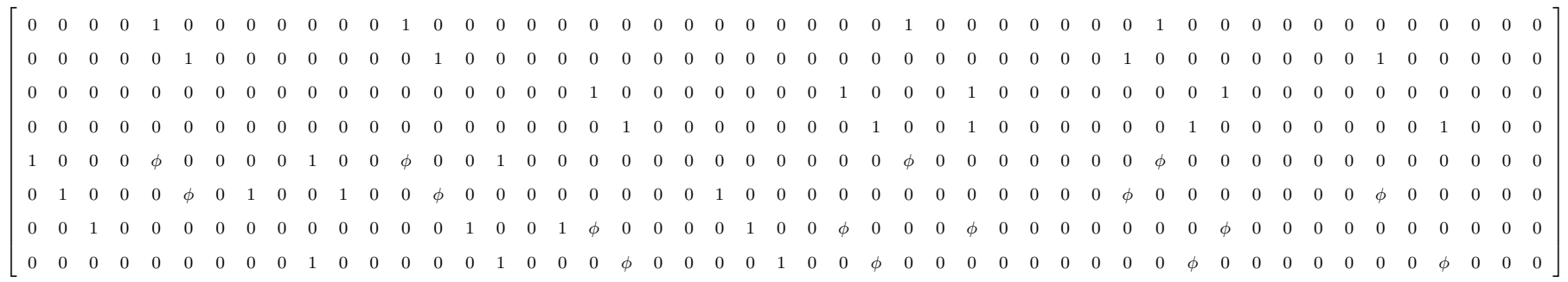

where $\phi=\phi_{1}+\phi_{2}$. Regardless of the value of $\phi_{1}$ and $\phi_{2}, J_{2}$ has rank 8 . This follows from the fact that each of the eight rows of $J_{2}$ has one in a different column. 
Then $B_{0}=J_{1} \Omega^{\frac{1}{2}}$ and $B_{2}=J_{2}\left(\Omega^{\frac{1}{2}} \otimes \Omega^{\frac{1}{2}}\right)$. Because the third moments of a standard normal random vector are zero,

$$
J=\left[\begin{array}{cc}
S_{0}^{\prime} B_{0} B_{0}^{\prime} S_{0} & 0_{4 \times 3} \\
0_{3 \times 4} & S_{1}^{\prime} B_{1} E\left(Z Z^{\prime} \otimes Z Z^{\prime}\right) B_{1}^{\prime} S_{1}
\end{array}\right] .
$$

Because $S_{0}^{\prime} B_{0} B_{0}^{\prime} S_{0}$ is a diagonal matrix with nonzero diagonal elements by the definition of $S_{0}$ and because $S$ is orthonormal and $B_{1}$ has full rank, $J$ is nonsingular.

\section{Acknowledgements}

We thank Mathias Trabandt for providing access to the data and code used in Christiano et al. (2011). We also have benefitted from helpful discussions with Frank Schorfheide, Silvia Gonçalves, Sophocles Mavroeidis, and from comments by the associate editor and by two referees. The views expressed in this paper are those of the authors and do not necessarily reflect those of the Federal Reserve Bank of Philadelphia or of the Federal Reserve System. 


\section{References}

1. Altig, D., L.J. Christiano, M. Eichenbaum and J. Lindé (2011), "Firm-Specific Capital, Nominal Rigidities and the Business Cycle," Review of Economic Dynamics 14, $225-247$.

2. Andrews, D.W.K. (1987), "Asymptotic Results for Generalized Wald Tests," Econometric Theory, 3, 348-358.

3. Andrews, I. and A. Mikusheva (2015), "Maximum Likelihood Inference in Weakly Identified Models," Quantitative Economics, 6, 123-152.

4. Antoine, B., and E. Renault (2012), "Efficient Minimum Distance Estimation with Multiple Rates of Convergence," Journal of Econometrics, 1709, 350-367.

5. Bao, Y. (2007), "The Approximate Moments of the Least Squares Estimator for the Stationary Autoregressive Model Under a General Error Distribution," Econometric Theory, 23, 1013-1021.

6. Bao, Y., and A. Ullah (2007), "The Second-Order Bias and Mean Squared Error of Estimators in Time Series Models," Journal of Econometrics, 140, 650-669.

7. Boivin, J., and M. Giannoni (2006), "Has Monetary Policy Become More Effective?", Review of Economics and Statistics, 88, 445-462.

8. Canova, F., and L. Sala (2009), "Back to Square One: Identification Issues in DSGE Models," Journal of Monetary Economics, 56, 431-449.

9. Chauduri, S., and E. Zivot (2011), "A New Method of Projection-Based Inference in GMM with Weakly Identified Nuisance Parameters," Journal of Econometrics, 164, 239-251.

10. Cheng, G., and J.Z. Huang, (2010), "Bootstrap Consistency for General Semiparametric M-Estimation," Annals of Statistics, 38, 2884-2915. 
11. Chernozhukov, V., and H. Hong (2003), "An MCMC Approach to Classical Estimation," Journal of Econometrics, 196, 293-346.

12. Christiano, L.J., M. Eichenbaum and C. Evans (2005), "Nominal Rigidities and the Dynamic Effects of a Shock to Monetary Policy," Journal of Political Economy, 113, $1-45$.

13. Christiano, L.J., M. Eichenbaum and M. Trabandt (2015), "Unemployment and Business Cycles," forthcoming: Econometrica.

14. Christiano, L.J., M. Eichenbaum and R.J. Vigfusson (2006), "Assessing Structural VARs," Macroeconomics Annual 2006, Acemoglu, D., K. Rogoff and M. Woodford (eds.), MIT Press, Boston, MA.

15. Christiano, L.J., M. Trabandt and K. Walentin (2011), "DSGE Models for Monetary Policy Analysis," in: Friedman, B.M. and M. Woodford (eds.): Handbook of Monetary Economics, Volume 3A, North-Holland, Amsterdam.

16. DiCecio, R. (2009), "Sticky Wages and Sectoral Labor Comovement", Journal of Economic Dynamics and Control, 33, 538-553.

17. DiCecio, R., and E. Nelson (2007), "An Estimated DSGE Model for the United Kingdom", Federal Reserve Bank of St. Louis Review, 89, 215-231.

18. Dovonon, P., and S. Gonçalves (2014), "Bootstrapping the GMM Overidentification Test under First-Order Underidentification," manuscript, Concordia University and University of Montreal.

19. Dridi, R., A. Guay, and E. Renault (2007), "Indirect Inference and Calibration of Dynamic Stochastic General Equilibrium Models", Journal of Econometrics, 136, $397-430$. 
20. Dufour, J.M., L. Khalaf, and M. Kichian (2013), "Identification-Robust Analysis of DSGE and Structural Macroeconomic Models," Journal of Monetary Economics, 60, 340-350.

21. Dufour, J.M., and M. Taamouti (2005), "Projection-Based Statistical Inference in Linear Structural Models with Possibly Weak Instruments," Econometrica, 73, 13511365.

22. Dufour, J.M., and P. Valery (2015), "Wald-type Tests When the Rank Conditions Fail: A Smooth Regularization Approach," manuscript, McGill University and HEC Montreal.

23. Dupor, B., J. Han, and Y.C. Tsai (2007), "What Do Technology Shocks Tell Us about the New Keynesian Paradigm?", Journal of Monetary Economics 56, 560-569.

24. Fernandez-Villaverde, J., J.F. Rubio-Ramirez, T.J. Sargent and M.W. Watson (2007), "ABCs (and Ds) of Understanding VARs," American Economic Review, 97, 10211026.

25. Guerron-Quintana, P., A. Inoue and L. Kilian (2013), "Frequentist Inference in Weakly Identified DSGE Models," Quantitative Economics, 4, 197-227.

26. Hall, P. (1992), The Bootstrap and Edgeworth Expansion, Springer: New York.

27. Hall, P., and J.L. Horowitz (1996), "Bootstrap Critical Values for Tests Based on Generalized-Method-of-Moments Estimators," Econometrica, 64, 891-916.

28. Hall, A.R., A. Inoue, J.M. Nason, and B. Rossi (2012), "Information Criteria for Impulse Response Function Matching Estimation of DSGE Models," Journal of Econometrics, 170, 499-518.

29. Iacoviello, M. (2005), "House Prices, Borrowing Constraints and Monetary Policy in the Business Cycle," American Economic Review, 95, 739-764. 
30. Inoue, A. and L. Kilian (2002), "Bootstrapping Smooth Functions of Slope Parameters and Innovation Variances in $\operatorname{VAR}(\infty)$ Models," International Economic Review, 43, 309-332.

31. Inoue, A., and L. Kilian (2016), "Joint Confidence Sets for Structural Impulse Responses," Journal of Econometrics 192, 421-342.

32. Jordà, Ò., and S. Kozicki (2011), "Estimation and Inference by the Method of Projection Minimum Distance," International Economic Review, 52, 461-487.

33. Kim, J.Y. (2002), "Limited Information Likelihood and Bayesian Analysis," Journal of Econometrics, 107, 175-193.

34. Klenow, P. J. and O. Kryvtsov (2008), "State-Dependent or Time-Dependent Pricing: Does It Matter for Recent U.S. Inflation?," Quarterly Journal of Economics, 123, 863904.

35. Kormilitsina, A., and D. Nekipelov (2016), "Consistent Variance of the Laplace Type Estimators: Application to DSGE Models," International Economic Review 57, 603622.

36. Liu, Z., D.F. Waggoner, and T. Zha (2011), "Sources of Macroeconomic Fluctuations: A Regime-Switching DSGE Approach," Quantitative Economics, 2, 251-304.

37. Lütkepohl, H. (1990), "Asymptotic Distributions of Impulse Response Functions and Forecast Error Variance Decompositions of Vector Autoregressive Models," Review of Economics and Statistics, 72, 116-25.

38. Magnus, J.R., and H. Neudecker (1999), Matrix Differential Calculus with Applications in Statistics and Econometrics, Revised Edition, Wiley, Chichester.

39. Nakamura, E. and J. Steinsson (2008), "Five Facts about Prices: A Reevaluation of Menu Cost Models," Quarterly Journal of Economics, 123, 1415-1464. 
40. Newey, W.K. and D. McFadden (1994), "Large Sample Estimation and Hypothesis Testing", in: R.F. Engle and D.L. McFadden, Handbook of Econometrics, Vol. 4, Elsevier.

41. Newey, W.K., and R.J. Smith (2004), "Higher Order Properties of GMM and Generalized Empirical Likelihood Estimators," Econometrica, 72, 219-255.

42. Phillips, P.C.B. (1989), "Partially Identified Econometric Models," Econometric Theory, $5,181-240$.

43. Qu, Z. (2014), "Inference in Dynamic Stochastic General Equilibrium Models with Possible Weak Identification," Quantitative Economics, 5, 457-494.

44. Rotemberg, J. and M.W. Woodford (1997), "An Optimization-Based Econometric Framework for the Evaluation of Monetary Policy", in: Bernanke, B., and J. Rotemberg (eds.), NBER Macroeconomics Annual, MIT Press, Cambridge, MA.

45. Sims, C.A., J.H. Stock and M.W. Watson (1990), "Inference in Linear Time Series Models with Some Unit Roots," Econometrica, 58, 113-144.

46. Smets, F. and R. Wouters (2007), "Shocks and Frictions in U.S. Business Cycles: A Bayesian Approach," American Economic Review 97, 586-606.

47. Stock, J.H., and J.H. Wright (2000), "GMM with Weak Identification," Econometrica, 68, 1055-1096.

48. Uribe, M., and V.Z. Yue (2006), "Country Spreads and Emerging Markets: Who Drives Whom?", Journal of International Economics 69, 6-36.

49. Woodford, M. (2003), Interest and Prices: Foundations of a Theory of Monetary Policy. Princeton University Press, Princeton, NJ. 
Table 1: Bias, Standard Deviation and RMSE of the Parameter $a$ in the Small-Scale New Keynesian Model

\begin{tabular}{|c|c|c|c|c|c|c|c|c|}
\hline \multirow[b]{2}{*}{$T$} & \multirow[b]{2}{*}{$p$} & \multirow[b]{2}{*}{$H$} & \multicolumn{3}{|c|}{ Diagonal Weighting Matrix } & \multicolumn{3}{|c|}{ Optimal Weighting Matrix } \\
\hline & & & $\begin{array}{l}\text { Mean } \\
\text { Bias }\end{array}$ & $\begin{array}{l}\text { Standard } \\
\text { Deviation }\end{array}$ & RMSE & $\begin{array}{l}\text { Mean } \\
\text { Bias }\end{array}$ & $\begin{array}{l}\text { Standard } \\
\text { Deviation }\end{array}$ & RMSE \\
\hline 100 & 2 & 2 & 0.0042 & 0.0150 & 0.0155 & 0.0022 & 0.0115 & 0.0117 \\
\hline 100 & 2 & 4 & 0.0062 & 0.0176 & 0.0186 & 0.0021 & 0.0116 & 0.0118 \\
\hline 100 & 2 & 8 & 0.0097 & 0.0206 & 0.0227 & 0.0031 & 0.0121 & 0.0125 \\
\hline 100 & 4 & 2 & 0.0064 & 0.0151 & 0.0164 & 0.0042 & 0.0119 & 0.0126 \\
\hline 100 & 4 & 4 & 0.0081 & 0.0171 & 0.0189 & 0.0044 & 0.0119 & 0.0126 \\
\hline 100 & 4 & 8 & 0.0113 & 0.0198 & 0.0228 & 0.0046 & 0.0123 & 0.0131 \\
\hline 100 & 6 & 2 & 0.0085 & 0.0155 & 0.0177 & 0.0061 & 0.0122 & 0.0136 \\
\hline 100 & 6 & 4 & 0.0102 & 0.0173 & 0.0200 & 0.0060 & 0.0122 & 0.0136 \\
\hline 100 & 6 & 8 & 0.0128 & 0.0197 & 0.0235 & 0.0064 & 0.0125 & 0.0140 \\
\hline 232 & 2 & 2 & 0.0022 & 0.0103 & 0.0105 & 0.0012 & 0.0073 & 0.0074 \\
\hline 232 & 2 & 4 & 0.0030 & 0.0122 & 0.0126 & 0.0011 & 0.0073 & 0.0074 \\
\hline 232 & 2 & 8 & 0.0043 & 0.0145 & 0.0151 & 0.0015 & 0.0076 & 0.0078 \\
\hline 232 & 4 & 2 & 0.0029 & 0.0100 & 0.0104 & 0.0020 & 0.0075 & 0.0078 \\
\hline 232 & 4 & 4 & 0.0034 & 0.0115 & 0.0120 & 0.0021 & 0.0074 & 0.0077 \\
\hline 232 & 4 & 8 & 0.0045 & 0.0137 & 0.0144 & 0.0021 & 0.0075 & 0.0078 \\
\hline 232 & 6 & 2 & 0.0038 & 0.0100 & 0.0107 & 0.0028 & 0.0076 & 0.0081 \\
\hline 232 & 6 & 4 & 0.0044 & 0.0113 & 0.0121 & 0.0028 & 0.0076 & 0.0081 \\
\hline 232 & 6 & 8 & 0.0053 & 0.0132 & 0.0143 & 0.0028 & 0.0077 & 0.0081 \\
\hline
\end{tabular}

Notes: $T$ denotes the sample size, $p$ the VAR lag order, and $H$ the maximum horizon of the impulse response functions. $a$ is the probability of a firm not adjusting its price. 
Table 2: Effective Coverage Probabilities of $90 \%$ Confidence Intervals for the Parameter $a$ in the Small-Scale New Keynesian Model

\begin{tabular}{lll|ll|ll}
\hline \hline & & & \multicolumn{2}{|c|}{ VAR Bootstrap } & \multicolumn{2}{c}{ DSGE Bootstrap } \\
\cline { 4 - 7 }$T$ & $p$ & $H$ & Diagonal & Optimal & Diagonal & Optimal \\
\hline 100 & 2 & 2 & 84.4 & 83.6 & 89.0 & 90.2 \\
100 & 2 & 4 & 82.0 & 68.4 & 88.8 & 90.2 \\
100 & 2 & 8 & 76.8 & 57.0 & 86.6 & 88.8 \\
100 & 4 & 2 & 84.2 & 81.6 & 87.8 & 89.6 \\
100 & 4 & 4 & 83.2 & 77.8 & 86.8 & 88.6 \\
100 & 4 & 8 & 81.0 & 58.4 & 86.0 & 87.8 \\
100 & 6 & 2 & 80.8 & 78.4 & 86.6 & 88.8 \\
100 & 6 & 4 & 78.8 & 74.2 & 85.8 & 87.8 \\
100 & 6 & 8 & 73.2 & 58.8 & 84.6 & 86.8 \\
232 & 2 & 2 & 86.4 & 84.6 & 87.2 & 89.6 \\
232 & 2 & 4 & 85.8 & 75.8 & 87.8 & 91.2 \\
232 & 2 & 8 & 87.0 & 67.6 & 86.4 & 90.2 \\
232 & 4 & 2 & 86.2 & 87.6 & 88.0 & 88.6 \\
232 & 4 & 4 & 85.6 & 87.0 & 87.0 & 88.8 \\
232 & 4 & 8 & 87.0 & 74.8 & 86.2 & 89.4 \\
232 & 6 & 2 & 88.0 & 86.2 & 89.0 & 89.0 \\
232 & 6 & 4 & 85.6 & 87.8 & 87.0 & 90.2 \\
232 & 6 & 8 & 87.0 & 81.6 & 86.8 & 87.4 \\
464 & 2 & 2 & 88.6 & 89.2 & 91.8 & 88.8 \\
464 & 2 & 4 & 90.6 & 85.0 & 91.0 & 88.8 \\
464 & 2 & 8 & 89.6 & 77.6 & 91.2 & 88.2 \\
464 & 4 & 2 & 88.8 & 89.0 & 91.2 & 90.4 \\
464 & 4 & 4 & 89.2 & 86.0 & 90.4 & 89.8 \\
464 & 4 & 8 & 88.6 & 82.4 & 91.8 & 89.6 \\
464 & 6 & 2 & 87.2 & 86.2 & 90.6 & 90.4 \\
464 & 6 & 4 & 89.8 & 86.4 & 90.6 & 89.4 \\
464 & 6 & 8 & 87.6 & 83.6 & 91.4 & 90.2 \\
\hline & & & & &
\end{tabular}

Notes: $T$ denotes the sample size, $p$ the VAR lag order, and $H$ the maximum horizon of the impulse response functions. $a$ is the probability of a firm not adjusting its price. 
Table 3: Effective Coverage Probabilities of 90\% Confidence Intervals for the Parameter a in the Small-Scale New Keynesian Model

\begin{tabular}{lll|ll}
\hline \hline$T$ & $p$ & $H$ & VAR Bootstrap & DSGE Bootstrap \\
\hline 100 & 2 & 2 & 90.2 & 92.6 \\
100 & 2 & 4 & 87.2 & 92.4 \\
100 & 2 & 8 & 85.6 & 91.6 \\
100 & 4 & 2 & 91.0 & 91.8 \\
100 & 4 & 4 & 91.4 & 92.0 \\
100 & 4 & 8 & 88.6 & 92.4 \\
100 & 6 & 2 & 96.4 & 93.4 \\
100 & 6 & 4 & 95.2 & 92.0 \\
100 & 6 & 8 & 94.2 & 91.0 \\
232 & 2 & 2 & 92.2 & 90.6 \\
232 & 2 & 4 & 91.6 & 91.8 \\
232 & 2 & 8 & 90.0 & 91.8 \\
232 & 4 & 2 & 91.8 & 91.4 \\
232 & 4 & 4 & 92.0 & 90.4 \\
232 & 4 & 8 & 90.0 & 91.8 \\
232 & 6 & 2 & 93.0 & 90.0 \\
232 & 6 & 4 & 92.8 & 89.0 \\
232 & 6 & 8 & 92.8 & 91.2 \\
\hline
\end{tabular}

Notes: $T$ denotes the sample size, $p$ the VAR lag order, and $H$ the maximum horizon of the impulse response functions. $a$ is the probability of a firm not adjusting its price. 


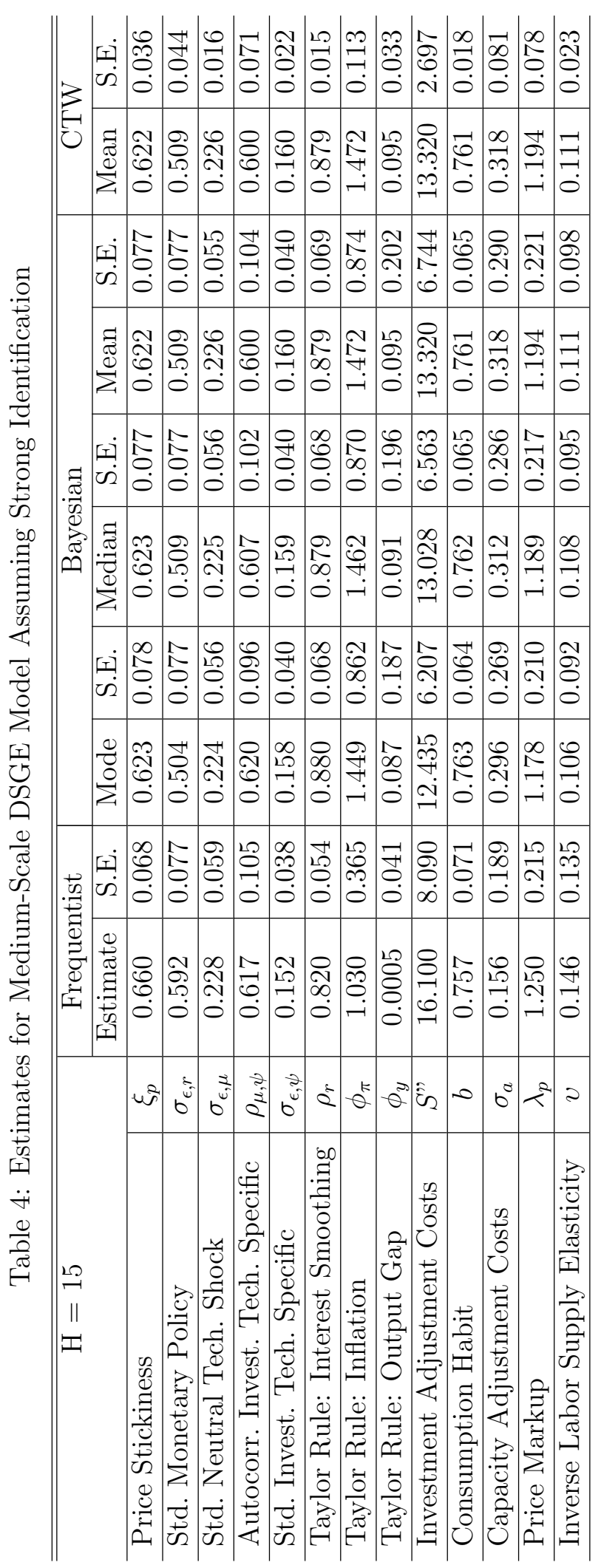

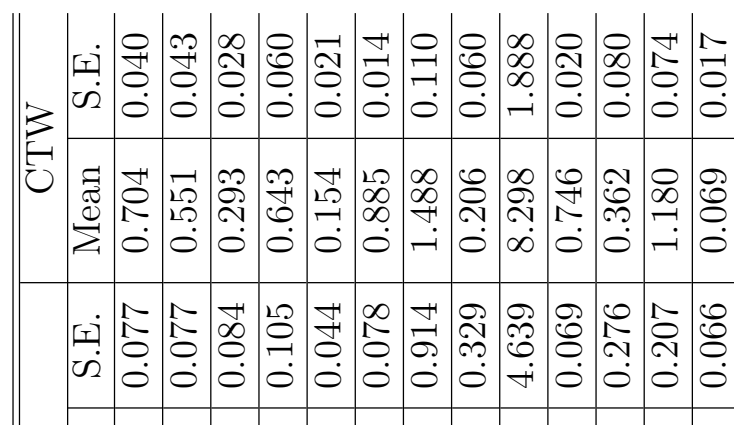

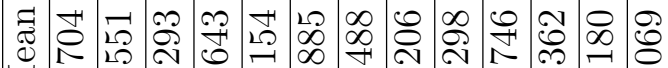

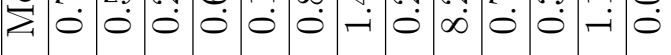

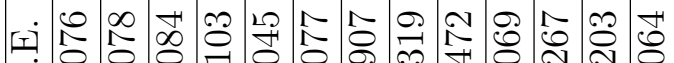

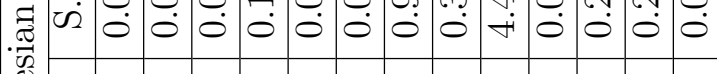

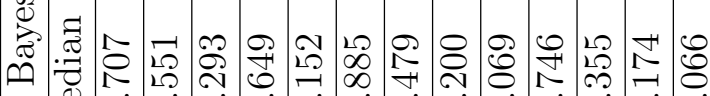

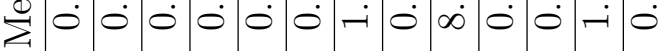

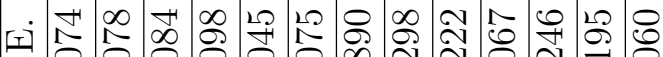

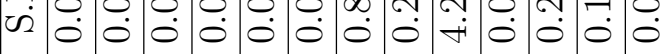

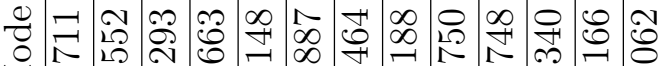

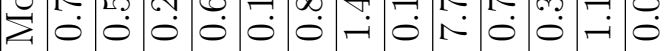

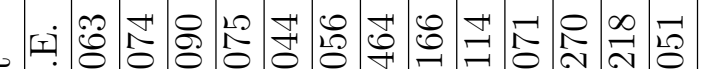

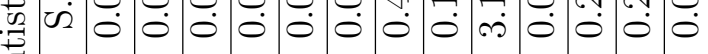
:

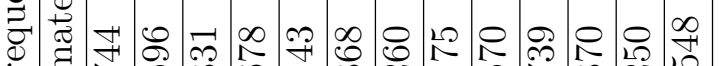

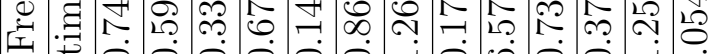

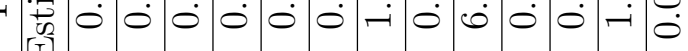

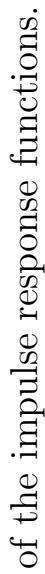
य人民

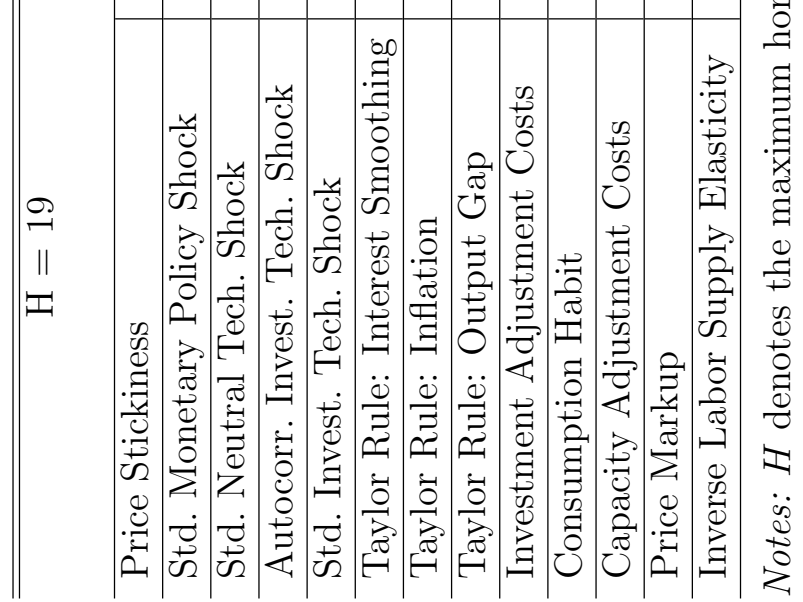


Table 5: 95\% Confidence Intervals for Medium-Scale DSGE Model Allowing for Weak Identification

\begin{tabular}{|l|c|cc|cc|}
\hline \hline \multicolumn{2}{|c|}{} & \multicolumn{2}{|c|}{$H=15$} & \multicolumn{2}{c|}{$H=19$} \\
\hline Price Stickiness & $\xi_{p}$ & 0.460 & 0.726 & 0.579 & 0.796 \\
\hline Std. Monetary Policy Shock & $\sigma_{\epsilon, r}$ & 0.380 & 0.664 & 0.385 & 0.690 \\
\hline Std. Neutral Tech. Shock & $\sigma_{\epsilon, \mu}$ & 0.182 & 0.299 & 0.211 & 0.374 \\
\hline Autocorr. Invest. Tech. Shock & $\rho_{\mu, \psi}$ & 0.330 & 0.764 & 0.437 & 0.792 \\
\hline Std. Invest. Tech. Shock & $\sigma_{\epsilon, \psi}$ & 0.108 & 0.231 & 0.101 & 0.239 \\
\hline Taylor Rule: Interest Smoothing & $\rho_{r}$ & 0.840 & 0.922 & 0.841 & 0.921 \\
\hline Taylor Rule: Inflation & $\phi_{\pi}$ & 1.210 & 1.912 & 1.241 & 1.915 \\
\hline Taylor Rule: Output Gap & $\phi_{y}$ & 0.018 & 0.262 & 0.063 & 0.425 \\
\hline Investment Adjustment Costs & $S^{\prime \prime}$ & 6.902 & 26.245 & 4.327 & 18.819 \\
\hline Consumption Habit & $b$ & 0.702 & 0.815 & 0.681 & 0.811 \\
\hline Capacity Adjustment Costs & $\sigma_{a}$ & 0.078 & 0.683 & 0.155 & 0.632 \\
\hline Price Markup & $\lambda_{p}$ & 1.017 & 1.502 & 1.015 & 1.464 \\
\hline Inverse Labor Supply Elasticity & $v$ & 0.056 & 0.227 & 0.027 & 0.162 \\
\hline
\end{tabular}

Notes: $H$ denotes the maximum horizon of the impulse response functions. 